\title{
Observing Hydrogen in Steel using Cryogenic Atom Probe Tomography: A Simplified Approach
}

\author{
Yi-Sheng Chen ${ }^{1,2, *}$, Paul A.J. Bagot ${ }^{1}$, Michael P. Moody ${ }^{1}$, Daniel Haley ${ }^{1}$ \\ ${ }^{1}$ Department of Materials, University of Oxford, Oxford OX1 3PH, UK \\ ${ }^{2}$ Australian Centre for Microscopy and Microanalysis, The University of Sydney, Sydney, NSW \\ 2006, Australia \\ *Corresponding author: yi-sheng.chen@sydney.edu.au
}

\begin{abstract}
This work demonstrates a new method to enable cryogenic atom probe tomography (cryoAPT) for the investigation of hydrogen in a high-strength steel, specifically to detect hydrogen localised to $\mathrm{V}-\mathrm{Mo}-\mathrm{Nb}$ carbides finely dispersed in the matrix. Prior cryogenic experiments required highly customised atom probe instrumentation to enable samples to be kept at cryogenic temperatures throughout the vacuum transfer process. Here we use an alternative approach without modification of the atom probe instrument itself, whilst still achieving hydrogen mapping. Additionally, we use this method to investigate the roles of solvent and solutes within the charging electrolyte, and we demonstrate that deuterated solute is not required when using heavy water as solvent, expanding the range of electrolytes that can be utilised in APT hydrogen charging experiments. This work reduces the experimental requirements for cryo-APT and makes the technique accessible to all APT equipped laboratories.
\end{abstract}




\section{Introduction}

Hydrogen embrittlement (HE) in steels is a major challenge in a wide variety of high-strength applications, such as in automotive components [1], bearings [2] and pressurised water reactors in nuclear power plants [3]. In all these systems, HE can cause catastrophic early component failure. Although the exact mechanisms that underpin HE are still a subject of significant debate [4-7], an existing strategy to mitigate this problem is by the incorporation of hydrogen 'traps' within steel microstructures. These traps obstruct the movement of atomic hydrogen toward regions of the microstructure most susceptible to the effects of HE [8-14]. The ability to trap hydrogen within a material provides a level of intrinsic HE resistance which can be further enhanced by a number of extrinsic approaches, e.g. hydrogen bake-out to remove hydrogen pre-service or the application of either a coating $[2,8]$ or surface strain [15-17] as a hydrogen ingress barrier. However, to both improve the effectiveness of these strategies and broaden their industrial applications, a better understanding of the atomic-scale mechanisms driving hydrogen trapping in steel is of great importance.

Hydrogen is the smallest interstitial solute atom able to diffuse near-freely within the lattice, and can be trapped at various microstructural features, such as dislocations, voids, vacancies and precipitates [18]. For example, the incorporation of finely-dispersed transition metal carbides, e.g. TiC or VC at nano-scale $(10-20 \mathrm{~nm})$, has been found to simultaneously provide strength and be beneficial to HE resistance in steels $[8,9,19,20]$. However, current atomic-scale understanding of how hydrogen is trapped by such carbides is still limited, where from an experimental perspective there is a lack of suitable protocols for nanoscale hydrogen mapping [21]. Accordingly, further experimental methodology to directly observe the hydrogen trapping in the carbides is required. 
Atom probe tomography (APT), with its high spatial resolution in combination with good chemical sensitivity, has been used for the observation of atomic hydrogen [22-28]. Whilst key to providing atom-scale imaging data on hydrogen, the limited field of view (typically $80 \times 80 \times 200$ $\mathrm{nm})$, and resolution $(\sim 1 \mathrm{~nm})$ implies that the method is best suited as a high-resolution method to complement other techniques. This is key to fully understanding the effect of HE, where information such as strain data may be critical [15-17]. However, two significant limitations in the use of APT for hydrogen imaging remain, namely the ambiguity between the signal from hydrogen genuinely within the material and that within the environment of the vacuum chamber [29, 30], and secondly there can be hydrogen signal loss due to the extremely fast diffusion of hydrogen during sample transfer. The former can be overcome, to some extent, by labelling with deuterium (D), which can be near-unambiguously distinguished from environmental hydrogen introduced during the analysis [22, 24-27]. The latter issue can be addressed by adapting cryogenic sample transfer protocols (cryo-transfer) to suppress hydrogen/deuterium diffusion loss [22, 24-26]. Although these experimental requirements are clear, there only a handful of successful APT studies of this kind, potentially as the required instrumentation is not available to most atom probe laboratories. For example, Takahashi et al. developed a modified 3DAP (Oxford Nanoscience) instrument to enable the first observation of hydrogen trapping by $\mathrm{TiC}$ via the use of a special gaseous charging cell together with a customised cryo-transfer chain [22]. Also, on a modified LEAP4000 XR (CAMECA), the authors' recent work applied a simple ex-situ liquid charging approach to achieve a comparable outcome of hydrogen mapping [24]. More recently, a customised protocol using a high vacuum device was demonstrated to achieve cryo-transfer by Stevenson et al. [31]. However, these instrument modification requirements are only feasible for a small number of APT facilities. 
In this work, alternate cryo-transfer protocols are developed to achieve cryo-APT without any instrument modification, enabling the investigation of hydrogen trapping in steel. As our protocol is conducted at atmosphere, ice contamination from environmental moisture during transfer remains problematic. To solve this, an in-situ sublimation procedure within the atom probe 'buffer' vacuum chamber is used to remove sample surface contamination before commencing APT analysis.

Additionally, to demonstrate the utility of the developed cryo-transfer, we investigate the respective roles of solute and solvent for the hydrogen uptake in an electrolytic hydrogen charging process via comparison experiments for the solute/solvent containing light and heavy hydrogen. Finally, to compare the behaviours of hydrogen localisation between protium $\left({ }^{1} \mathrm{H}\right)$ and deuterium, the distribution of ${ }^{1} \mathrm{H}$ is examined in this work.

\section{Material and Methods}

\subsection{Sample steel and precipitate analyses in TEM and APT}

A ferritic steel designed to incorporate a high number density of $\mathrm{V}-\mathrm{Mo}-\mathrm{Nb}$ transition metal carbides was used in this study (as per [24]) with the nominal chemical composition given in Table 1. The material sheet was manufactured in a continuous production from $980^{\circ} \mathrm{C}$, hot rolling and then coiling at $650^{\circ} \mathrm{C}$, which typically allows the formation of high-density carbide precipitates [32]. The yield strength, tensile strength, stiffness and Poisson ratio for the as-received material were 820 and $897 \mathrm{MPa}, 200 \mathrm{GPa}$ and 0.3 respectively. The material sheet was cut and mechanically thinned into matchstick bars of 1 x 1 x $20 \mathrm{~mm}$, followed by APT needle sample preparation using a standard two-stage electropolishing process at $10-30 \mathrm{~V}$ with $25 \%$ perchloric acid in acetic acid and $2 \%$ perchloric acid in butoxyethanol for the first and second stages, respectively [33]. APT 
experiments were conducted in voltage-pulsed mode, using a LEAP 3000X HR (CAMECA) with $15 \%$ pulse fraction, a pulse frequency of $200 \mathrm{kHz}$ and sample stage temperature of $60 \mathrm{~K}$. These parameters were selected based on previous studies for their influence on the chemical accuracy of APT measurement [34-36]. Data was finally analysed using IVAS (Version 3.6.12, CAMECA).

Table 1. Nominal and atom-probe-measured compositions of the studied material. All compositions balance with iron. The corresponding 3D atom maps are shown in Figure 1.

\begin{tabular}{ccccccccccc}
\hline composition & Mn & Mo & $\mathbf{V}$ & $\mathbf{C}$ & $\mathbf{N b}$ & $\mathbf{A l}$ & $\mathbf{S i}$ & $\mathbf{N}$ & $\mathbf{P}$ & $\mathbf{C r}$ \\
\hline Nominal (wt.\%) & 1.56 & 0.51 & 0.25 & 0.096 & 0.056 & 0.05 & 0.026 & 0.0038 & - & - \\
Nominal (at.\%) & 1.59 & 0.3 & 0.27 & 0.45 & 0.034 & 0.1 & 0.05 & 0.02 & - & - \\
Measured (at.\%) & 1.39 & 0.32 & 0.3 & 0.46 & 0.034 & 0.12 & 0.05 & 0.02 & 0.019 & 0.016 \\
\hline
\end{tabular}

The APT mass spectrum and reconstructed 3D atom maps (reconstruction per method of Bas et al. [37]) of the as-received sample are shown in Figure 1(a) and (b), respectively, with the measured composition shown in Table 1 after using a global background subtraction. The obtained composition generally agrees with the nominal composition, except for the detection of $\mathrm{P}$ and $\mathrm{Cr}$, present as common impurities from material production [38], and a moderate depletion of $\mathrm{Mn}$, potentially due to field-of-view effects [39]. The mass spectrum in Figure 1(a) shows all the peak assignments for ionic identification for the composition measurements [39]. The deconvolution of $\mathrm{C}_{2}{ }^{+}$and $\mathrm{C}_{4}{ }^{2+}$ contributions for the 24 Da peak was undertaken based upon the measured amount of 24.5 Da peak for $\left({ }^{12} \mathrm{C}_{3}{ }^{13} \mathrm{C}\right)^{2+}$ (approx. 62 counts) and 25 Da peak for $\left({ }^{12} \mathrm{C}_{2}{ }^{13} \mathrm{C}_{2}\right){ }^{2+} /\left({ }^{12} \mathrm{C}^{13} \mathrm{C}\right)^{+}$ (approx. 67 counts) [35], where the contribution of $\left({ }^{12} \mathrm{C}_{2}{ }^{13} \mathrm{C}_{2}\right)^{2+}$ in 25 Da peak was accounted by the isotopic abundances of ${ }^{12} \mathrm{C}_{3}{ }^{13} \mathrm{C}(4.29 \%)$ and ${ }^{12} \mathrm{C}_{2}{ }^{13} \mathrm{C}_{2}$ (0.07\%) [40]. Also, the deconvolution of ${ }^{28} \mathrm{Si}^{2+}$ and ${ }^{14} \mathrm{~N}^{+}$contributions for the 14 Da peak was undertaken based upon the ${ }^{29} \mathrm{Si}^{2+}$ peak at 14.5 Da with isotopic abundance of silicon. Note that the background hydrogen peak at $1 \mathrm{Da}$, which was not incorporated in the composition measurement above but led to a content of 0.0037 at. $\%$ if accounted for. Lastly, at the 3D reconstruction stage using the principle from [37], an image compression factor (ICF) of 1.25 and a tip radius of $24 \mathrm{~nm}$ estimated by IVAS were applied after 
accounting the calibration suggested in [41] with the theoretical lattice spacing of (200) and (110) in body-centre-cubic iron, as shown in Figure S1 of the supplementary section.

In order to obtain the precipitate composition, a precipitate definition using the maximum separation method (details in [33]) against the nominal precipitate atoms, i.e. $\mathrm{V}$, Mo, $\mathrm{Nb}, \mathrm{C}, \mathrm{C}_{2}$, and $\mathrm{C}_{3}$, was applied with the parameters selected using the 'objective function' protocol by Williams et al. [42]. This analysis yields the cluster analysis parameters in the maximum separation method described in [33]. The key parameters defining this approach are $d_{\max }$ the maximum distance separating a solute atom and its nearest solute neighbour within the same cluster, $\mathrm{N}_{\min }$ the minimum number of atoms to be regarded as a cluster, the enveloping distance, $\mathrm{L}$, and erosion distance, $\mathrm{d}_{\text {erosion, }}$ which together enable the incorporation of matrix atoms into the clusters. In this study the values of these parameters were determined to be $1 \mathrm{~nm}, 100$ atoms, $0.5 \mathrm{~nm}$ and $0.5 \mathrm{~nm}$, respectively. This resulted in 27 defined precipitates within the 4 million incorporated ions, as shown in Figure S2 of the supplementary section. As local magnification artefacts in APT can erroneously lead to matrix elements being incorporated into the analysis of precipitates [33], only the precipitate ions, i.e. $\mathrm{V}, \mathrm{Mo}, \mathrm{Nb}, \mathrm{C}$, and $\mathrm{N}$, are accounted for in precipitate composition measurements, as shown in Table 2, where the deconvolutions of $\mathrm{C}$ and $\mathrm{N}$ peaks were undertaken similar to the global mass spectrum analysis. The elemental ratio of metal-to-non-metal (M:X) is 1.26:1 which suggests the carbide stoichiometry is between $\mathrm{M}_{4} \mathrm{X}_{3}$ and $\mathrm{MX}$ (having $\mathrm{M}: \mathrm{X}=1.33: 1$ and 1:1, respectively). Considering the uncertainty of carbon quantification in APT analyses [3436], this ratio could be as low as 1.21:1 if accounting for the worst-case of carbon deficiency of 4.7\% reported in the literature [34], however this does not alter the conclusion that $\mathrm{M}_{4} \mathrm{X}_{3}$ and $\mathrm{MX}$ are the only two likely possibilities for the carbide stoichiometry. 
Table 2. Precipitate composition of the as-received sample as measured by APT

\begin{tabular}{c|c|c|c|c|c}
\hline & V & Mo & Nb & C & N \\
\hline \multirow{2}{*}{ Count of atoms } & 7901 & 6492 & 1051 & 11758 & 470 \\
\cline { 2 - 6 } & \multicolumn{3}{|c|}{15445} & \multicolumn{2}{|c}{12228} \\
\hline Normalised to C + N & \multicolumn{3}{|c|}{1.26} & \multicolumn{3}{|c}{1} \\
\hline
\end{tabular}

\subsection{Hydrogen charging}

To introduce hydrogen into the material, the electropolished APT needle samples were electrolytically charged in either $\mathrm{D}_{2} \mathrm{O}+0.1 \mathrm{M} \mathrm{NaOD}$ (both from Sigma-Aldrich), $\mathrm{D}_{2} \mathrm{O}+0.1 \mathrm{M}$ $\mathrm{NaOH}$ or $\mathrm{H}_{2} \mathrm{O}+0.1 \mathrm{M} \mathrm{NaOD}$ at $2.2 \mathrm{~V}$ for 30 seconds in a 2-wire configuration, using a gold wire as the counter-electrode, as per our prior work [24]. After the charging was completed, the samples were immediately removed from the charging cell and mounted to an APT 'puck' holder. The entire mounting procedure was completed within approximately 2 minutes before undertaking the cryo-transfers described in the next section.

\subsection{Sample transfers for APT experiments}

After hydrogen charging and sample mounting, three different types of APT transfer methods were investigated, each differentiated by the thermal and environmental conditions of the samples. The initial reference method was conducted at room temperature, hereafter referred to as 'RT Transfer', in which the puck with the charged samples on a puck carrier, or 'carousel', was immediately introduced into the atom probe sample loading chamber, or 'loadlock', for pumping down to a pressure of approximately $10^{-5} \mathrm{~Pa}$. The sample was then moved to the buffer vacuum chamber and finally the analysis chamber to begin the APT experiment, allowing 20 minutes to reduce the pressure to levels appropriate for loading into the analysis chamber. 
To cool the sample to very low temperature and thereby minimise the D diffusion, cryogenic freezing was employed prior to the proposed cryo-transfers, hereafter termed 'Direct Transfer' and 'Box Transfer' as shown in Figure 2(a) and (b), respectively. As per Figure 2(a), the Direct Transfer process involves removing the cryo-samples from $\mathrm{LN}_{2}$ and then immediately transferring them into the atom probe loadlock. However, this approach exposes the cryo-sample to atmospheric moisture leading to surface condensation. In comparison, the Box Transfer (Figure 2(b)) was developed to create a drier environment by adapting a custom glove box to limit the moisture supply when the sample was transferred from the $\mathrm{LN}_{2}$ container to the loadlock. The procedure for this transfer method is given in the supplementary section.

A thermally-insulating carousel 'cryo block' (CAMECA) as shown in Figure 2(c) consisting of PTFE (as shown in Figure 2(d)) insulation and an aluminium 'block' for thermal mass, was used for the cryo-transfers. An additional 'hat' was constructed to protect the APT samples and to further minimise moisture condensation. The Box Transfer method further used a 125-litre acrylic cubic box with one side connected to the LEAP loadlock through a 500-litre plastic bag for sample transfer. Within the box, a hygrometer (RH318, Omega) and $800 \mathrm{~g}$ of silica gel (Sigma-Aldrich) were also inserted respectively for humidity data acquisition and humidity minimisation within the box.

\subsection{Ice sublimation within atom probe}

The entire experimental procedure with the corresponding sample temperature at each stage is outlined in Figure 3(a). The sample was first charged with hydrogen at room temperature, then mounted to the puck and immediately cooled in $\mathrm{LN}_{2}$ as specified in steps (a) to (c). After the cryotransfer, the sample was loaded to the LEAP sample stage for specimen observation as shown in 
step (d) and (e). The images of as-loaded samples from the respective cryo-transfers are shown in Figure 3(b) where different levels of ice condensation can be seen. Owing to ice build-up during the initial stages of cooling and transfer, an ice-removal procedure was conducted by holding the samples with the LEAP transfer arm in the buffer chamber at approximately $10^{-6} \mathrm{~Pa}$ after removing the samples from the analysis chamber to slowly sublimate accumulated ice, as shown in Figure 3(c). This corresponds to an approximate sublimation temperature $\left(\mathrm{T}_{\text {sub }}\right)$, given in Figure 3(a), where the time required for the entire sublimation process was 60 and 50 minutes for the Direct Transfer and Box Transfer, respectively.

2.5 Determining sample temperature via pressure and heat transfer methods

After sublimation, the sample reached a temperature ' $\mathrm{T}_{\mathrm{end}}$ ' as highlighted in Figure 3(a), regarded as the highest temperature experienced by the sample in the entire procedure. $T_{\text {end }}$ can be estimated by either the above sublimation pressure or via the amplitude of the thermal response of the LEAP stage given by the temperature readout. To do so, the heat transfer between the sample at $\mathrm{T}_{\text {sample }}$ and the stage that results in a response temperature $\left(\mathrm{T}_{\mathrm{e}}\right)$ can be described in $\mathrm{Eq}(1)$ :

$$
T_{\text {sample }}=R\left(T_{e}-T_{\text {stage }}\right)+T_{\text {stage }}
$$

where $\mathrm{R}$ is a constant for the combined stage and puck assembly. After loading a sample at $293 \mathrm{~K}$ into the sample stage at $60 \mathrm{~K}, \mathrm{R}$ was found to be 22 by reading the maximum response temperature of the stage at $70.6 \mathrm{~K}$ with the inputs of $\mathrm{T}_{\text {sample, }} \mathrm{T}_{\text {stage }}$ and $\mathrm{T}_{\mathrm{e}}$ in $\mathrm{Eq}(1)$ as 293,60 and 70.6, respectively. Moreover, as shown in Figure 4, with multiple measurements of $\mathrm{T}_{\text {sample }}$ at various heating times combined with the known initial temperature prior to the sublimation, the functions of the sample temperature to the heating time for both methods can be obtained. This gives heating rates of 2.0 and $1.2 \mathrm{~K} /$ minute according to the measurements from the heat transfer method and 
pressure method, respectively. The heat transfer method was deemed as more accurate than the pressure measurement, which is subject to the gauge quality considerations. As such, the heat transfer method will be referred to hereafter for the determination of sample temperature in the following content.

\section{Results and discussion}

\subsection{Atom probe results}

All the APT experiments and their resulting ratios of global D to $\mathrm{V}$ counts (D/V) are listed in Table 3. The vanadium signal was used for normalisation here because it has relatively simple isotopic peaks at 25.5 Da $\left({ }^{51} \mathrm{~V}^{2+}\right)$ and $17 \mathrm{Da}\left({ }^{51} \mathrm{~V}^{3+}\right)$ which allow for a more straight-forward quantification as compared with other carbide elements. For the room-temperature transfer which was held for 20 minutes in vacuum, the sample has a D/V ratio of 0.0081 . After acquisition of this data, the experiment was stopped, and the sample was taken back to the buffer carousel at room temperature for 12 hours to completely desorb the charged D, which resulted in another data noted as 'overnight' in Table 3, giving a D/V ratio of 0.0073 . This is a measure of the noise signal within proximity of $2 \mathrm{Da}$, using the same ion labelling criteria in other D-incorporated datasets, and is used as the D-free reference in the content below.

Both the Box Transfer and Direct Transfer samples were sublimated for 50 and 60 minutes respectively, with associated maximum temperatures of $168 \mathrm{~K}$ and $189 \mathrm{~K}$, as per $\mathrm{Eq}(1)$. All the D/V data from the cryo-transfers are significantly higher than the overnight/D-free reference and are comparable to our previous work (D/V of 0.0388) using a commercial cryo-transfer protocol providing cryogenic treatment of the sample at $100 \mathrm{~K}$ [24]. The data hence suggests the presence of $\mathrm{D}$ in the samples. As D desorption from carbide traps is a thermally activated process [12], we 
expected that in the Direct Transfer samples, which experienced a higher sublimation temperature, should have desorbed more D prior to analysis than in the case of the Box Transfer samples. While the results shown in Table 3 appear not to support this, this is attributed to having insufficient data for robust error analysis to verify this hypothesis. Apart from the sample temperature, the amount of trapped hydrogen could also be a function of the configuration of the observed carbide. However, this is beyond the scope of this method development work and requires more systematic study to understand the exact factors.

Table 3. Sublimation times and associated sample temperatures from the sample transfer methods and their resulting ratios of global D counts to V counts. Vacuum cryogenic sample transfer (VCT) method from [24] is provided for comparison.

\begin{tabular}{lccc}
\hline \multicolumn{1}{c}{ Experiment } & Sublimation time (minute) & Sample temperature (K) & D/V ratio \\
\hline RT Transfer & - & 293 & 0.0081 \\
Overnight & - & 293 & 0.0073 \\
Direct Transfer & 60 & 189 & 0.0508 \\
Direct Transfer & 60 & 189 & 0.0473 \\
Box Transfer & 50 & 168 & 0.0382 \\
Box Transfer 2 & 50 & 168 & 0.0371 \\
\hline VCT (from Ref[24]) & - & 100 & 0.0388 \\
\hline
\end{tabular}

The 3D reconstructions of RT transfer and overnight desorption are shown in Figure 5(a) and (b), respectively, where carbide and D-clustering areas are delineated respectively by 1.5 at. $\%$ $\mathrm{V}$ isoconcentration surfaces (isosurfaces) in green and the 0.4 at. \% D isosurfaces in red. In Figure 5(a), a few D clusters that are spatially correlated to the carbides can be seen, which suggests the presence of hydrogen within the carbides in this material, whereas the absence of any D in Figure 5(b) (examined with same 0.4 at.\% D isosurface) indicates any D observed in (a) is now no longer observable. These results agree with the data shown in Table 3 demonstrating a lack of D retainment for the RT transfer. On the other hand, Figure 6(a) shows the D and V isosurfaces from the Direct Transfer experiment in Table 3, where their spatial correlation can be clearly seen. In Figure 7(a) from the Box Transfer experiment, the spatial correlations between the D and V isosurfaces can be observed, and two selected regions with D cluster incorporated are presented 
with sliced views as shown in (b) and (c), demonstrating the applicability of the proposed cryotransfer for hydrogen investigation. With only a few custom components, this approach removes the need for a dedicated instrument. The specific differences with the previous study [24] are summarised in Table 4:

Table 4. Comparison of the used equipment between the cryo-sample transfer methods of the current study and the previous [24]

\begin{tabular}{|c|c|c|}
\hline Purpose & Previous study [24] & Current study \\
\hline Sample cooling & $\begin{array}{c}\text { Cooling stage within a vacuum } \\
\text { chamber }\end{array}$ & $\begin{array}{c}\text { Direct immersion in an } \mathrm{LN}_{2} \\
\text { bath }\end{array}$ \\
\hline $\begin{array}{c}\text { Isolation of sample from environment } \\
\text { during loading }\end{array}$ & Via a commercialised cold chain & $\begin{array}{c}\text { Via a custom-developed } \\
\text { glove box }\end{array}$ \\
\hline $\begin{array}{c}\text { Maintaining cryogenic conditions in } \\
\text { vacuum }\end{array}$ & $\begin{array}{l}\text { Through a } \mathrm{LN}_{2} \text { attached cold } \\
\text { finger }\end{array}$ & Through a thermal mass \\
\hline
\end{tabular}

We believe further refinements are possible as the final observed concentration may have undergone considerable signal loss, potentially as hydrogen is theoretically mobile even at very low temperature [43].

In the proposed cryo-transfers, the lowest final sample temperature we achieved was $168 \mathrm{~K}$, largely due to the sublimation requirement, whereas $100 \mathrm{~K}$ was reported in [24] and $89 \mathrm{~K}$ by Stephenson et al. [31]. Nevertheless, the present protocols are straight-forward and require less sophisticated instrumentation to allow hydrogen observations. We believe, the protocols here should be useful for studies investigating hydrogen traps having similar or stronger hydrogen trapping abilities than for the type of carbide hydrogen traps here (either $\mathrm{M}_{4} \mathrm{X}_{3}$ or MX), e.g. TiC [8]. In addition to sample temperature, the present protocols do not allow the samples being transferred entirely within a controlled atmosphere, unlike our solutions which incorporate a shuttle/suitcase design [31, 44]. Such systems may be more amenable where there are concerns regarding interactions with atmospheric environments, e.g. oxidation. 


\subsection{Investigating the role of electrolyte in hydrogen liquid charging}

Using the transfer system above, we investigated the importance of solute and solvent in the charging solution for deuteration, showing the origin of hydrogen in the charged samples and the relative contribution of solute/solvent hydrogen. By comparing various isotopic combinations of hydrogen charging solutions, i.e. normal $\mathrm{H}_{2} \mathrm{O}+0.1 \mathrm{M} \mathrm{NaOH}$, fully deuterated $\mathrm{D}_{2} \mathrm{O}+0.1 \mathrm{M} \mathrm{NaOD}$, and the unconventional $\mathrm{D}_{2} \mathrm{O}+0.1 \mathrm{M} \mathrm{NaOH}$ and $\mathrm{H}_{2} \mathrm{O}+0.1 \mathrm{M} \mathrm{NaOD}$, the actual hydrogen source can be investigated by the resultant H/D detection. The resulting mass spectra, normalised to the corresponding vanadium peak intensities, from two experiments utilising unconventional charging solutions are shown in Figure 8(b) and (c), respectively, and compared with the data from previous studies using $\mathrm{D}_{2} \mathrm{O}+0.1 \mathrm{M} \mathrm{NaOD}$ in (a) and $\mathrm{H}_{2} \mathrm{O}+0.1 \mathrm{M} \mathrm{NaOH}$ in (d) [24].

In Figure 8(b) from using $\mathrm{NaOH}$ in $\mathrm{D}_{2} \mathrm{O}$, the $2 \mathrm{Da}$ peak is as significant as the data from $\mathrm{NaOD}$ in $\mathrm{D}_{2} \mathrm{O}$. Although the 2 Da peak in Figure 8(a) with the use of the previous protocol [24] seems lower than for the current protocol (Figure 8(b)), this is likely due to fluctuations in measured $\mathrm{H}$ content due to operational factors, such as variability in the time-to-cooling after hydrogen charging completion. On the other hand, hydrogen charging using $\mathrm{NaOD}$ in $\mathrm{H}_{2} \mathrm{O}$, as shown in (c), does not result in an observable peak at $2 \mathrm{Da}$. The 3D atom maps of the $\mathrm{D}_{2} \mathrm{O}+\mathrm{NaOH}$ data are presented in Figure 9, which also shows the result of a statistical nearest-neighbour analysis [33] in (b) highlighting the spatial association between D signal and the carbides. Sliced views in Figure 9 (c) and (d) show the positions of D and H, respectively, in relation to the carbides, represented by $\mathrm{V}$ atoms. These confirm the presence of $\mathrm{D}$ localisations in the carbides but not ${ }^{1} \mathrm{H}$. These results suggest the $0.1 \mathrm{M} \mathrm{NaOD} / \mathrm{NaOH}$ solute is not the primary origin of the observed localised hydrogen, implying that its use of is not critical to the charging process. This observation opens up the range of solutions that can be utilised beyond deuterium-based solutes. 
In the normalised mass spectra in Figure 8, the $1 \mathrm{Da}$ from $\mathrm{H}_{2} \mathrm{O}$ charged samples, i.e. (c) and (d), are both stronger than the 1 Da peaks of the $\mathrm{D}_{2} \mathrm{O}$ charged samples which should originate from the environmental hydrogen background, suggesting that the protium signals can be considerably introduced into the samples. To confirm the spatial distribution of protium, Figure 10 shows the $3 \mathrm{D}$ reconstructions of a $\mathrm{H}_{2} \mathrm{O}+0.1 \mathrm{M} \mathrm{NaOD}$ charged sample in (a), the result of ${ }^{1} \mathrm{H} / \mathrm{V}$ nearest neighbour analysis in (b) as well as the slice view with ${ }^{1} \mathrm{H}$ and $\mathrm{V}$ positions in (c) to present the associated spatial distributions. Additionally, the isosurfaces of ${ }^{1} \mathrm{H}$ and $\mathrm{V}$ from a $\mathrm{H}_{2} \mathrm{O}+0.1 \mathrm{M}$ $\mathrm{NaOH}$ charged sample reported in [24] are shown in Figure 10(d), suggesting the consistent protium localisations at carbide areas presented in this study. However, any future experiment using standard isotopic hydrogen sources needs to consider the high level of background hydrogen noise introduced by the APT experiment as shown at the 1 Da of Figure 8(a) and (b). Accordingly, deuteration for similar analyses is highly suggested when the goal is quantitative measurements of hydrogen trapping.

\section{Conclusions}

In this paper, a simplified cryo-transfer protocol enabling deuterium observation within a VMo-Nb carbide containing ferritic is demonstrated. The method successfully avoids considerable instrumentation enhancements used in previous works and demonstrates an approach to address the long-standing challenge with regards to ice contamination on the surface of APT cryo-samples and proposes a means by which the specimen temperature can be monitored during transfer. These techniques will more widely enable cryo-transfer experiments in conventionally equipped atom probe laboratories. Furthermore, the development of the presented protocols is targeted to improve understanding to the behaviour of hydrogen within metals and will provide further data for the design of hydrogen-embrittlement-resisting materials. 


\section{Acknowledgement}

This project was performed under the support of EPSRC under the HEmS Programme Grant EP/L014742/1. The authors thank the sample supply from Prof. W. Mark Rainforth of the

University of Sheffield. Y.-S. C. acknowledges the financial supports from Taiwan Ministry of Education, Prof. Julie Cairney of the University of Sydney and his family. The data can be accessed at: https://ora.ox.ac.uk/objects/uuid:6762addd-4f76-481a-8ea4-dcb9939b74c7

\section{References}

1. Okayasu, M. and Yang, L.L., Influence of microstructure on the mechanical properties and hydrogen embrittlement characteristics of 1800MPa grade hot-stamped 22MnB5 steel. Journal of Materials Science, 2019. 54(6): p. 5061-5073.

2. Stopher, M.A. and Rivera-Diaz-del-Castillo, P.E.J., Hydrogen embrittlement in bearing steels. Materials Science and Technology, 2016. 32(11): p. 1184-1193.

3. Toribio, J., Vergara, D., and Lorenzo, M., Role of in-service stress and strain fields on the hydrogen embrittlement of the pressure vessel constituent materials in a pressurized water reactor. Engineering Failure Analysis, 2017. 82: p. 458-465.

4. Robertson, I.M., et al., Hydrogen Embrittlement Understood. Metall. Mater. Trans. A, 2015. 46a(6): p. 2323-2341.

5. Lynch, S., Hydrogen embrittlement phenomena and mechanisms. Corrosion Reviews, 2012. 30(3-4): p. 105-123.

6. Nagumo, M., Hydrogen related failure of steels - a new aspect. Materials Science and Technology, 2004. 20(8): p. 940-950.

7. Barrera, O., et al., Understanding and mitigating hydrogen embrittlement of steels: $a$ review of experimental, modelling and design progress from atomistic to continuum. Journal of Materials Science, 2018. 53(9): p. 6251-6290.

8. Bhadeshia, H.K.D.H., Prevention of Hydrogen Embrittlement in Steels. I S I J International, 2016. 56(1): p. 24-36.

9. Li, L.F., et al., Effects of vanadium precipitates on hydrogen trapping efficiency and hydrogen induced cracking resistance in X80 pipeline steel. International Journal of Hydrogen Energy, 2018. 43(36): p. 17353-17363.

10. Pressouyre, G.M., Trap Theory of Hydrogen Embrittlement. Acta Metallurgica, 1980. 28(7): p. 895-911.

11. Pressouyre, G.M. and Bernstein, I.M., Nature and Behavior of Hydrogen Traps in IronAlloys. Jom-Journal of Metals, 1976. 28(12): p. A70-A70.

12. Choo, W.Y. and Lee, J.Y., Hydrogen Trapping Phenomena in Carbon-Steel. Journal of Materials Science, 1982. 17(7): p. 1930-1938. 
13. Wei, F.G. and Tsuzaki, K., Quantitative analysis on hydrogen trapping of TiC particles in steel. Metallurgical and Materials Transactions a-Physical Metallurgy and Materials Science, 2006. 37a(2): p. 331-353.

14. Turnbull, A., Perspectives on hydrogen uptake, diffusion and trapping. International Journal of Hydrogen Energy, 2015. 40(47): p. 16961-16970.

15. Li, X.F., et al., Effect of shot peening on hydrogen embrittlement of high strength steel. International Journal of Minerals Metallurgy and Materials, 2016. 23(6): p. 667-675.

16. Takakuwa, O. and Soyama, H., Preventing hydrogen embrittlement in stainless steel by means of compressive stress induced by cavitation peening. Journal of Engineering-Joe, 2015: p. 1-4.

17. Toribio, J., et al., Residual stress redistribution induced by fatigue in cold-drawn prestressing steel wires. Construction and Building Materials, 2016. 114: p. 317-322.

18. Pundt, A. and Kirchheim, R., Hydrogen in metals: Microstructural aspects. Annual Review of Materials Research, 2006. 36: p. 555-608.

19. Depover, T. and Verbeken, K., Evaluation of the effect of V4C3 precipitates on the hydrogen induced mechanical degradation in $\mathrm{Fe}-\mathrm{C}-\mathrm{V}$ alloys. Materials Science and Engineering a-Structural Materials Properties Microstructure and Processing, 2016. 675: p. 299-313.

20. Depover, T. and Verbeken, K., The effect of TiC on the hydrogen induced ductility loss and trapping behavior of Fe-C-Ti alloys. Corrosion Science, 2016. 112: p. 308-326.

21. Koyama, M., et al., Recent progress in microstructural hydrogen mapping in steels: quantification, kinetic analysis, and multi-scale characterisation. Materials Science and Technology, 2017. 33(13): p. 1481-1496.

22. Takahashi, J., et al., The first direct observation of hydrogen trapping sites in TiC precipitation-hardening steel through atom probe tomography. Scripta Materialia, 2010. 63(3): p. 261-264.

23. Zhu, X., et al., Hydrogen trapping sites and hydrogen-induced cracking in high strength quenching \& partitioning $(Q \& P)$ treated steel. International Journal of Hydrogen Energy, 2014. 39(24): p. 13031-13040.

24. Chen, Y.-S., et al., Direct observation of individual hydrogen atoms at trapping sites in a ferritic steel. Science, 2017. 355(6330): p. 1196-1199.

25. Takahashi, J., Kawakami, K., and Kobayashi, Y., Origin of hydrogen trapping site in vanadium carbide precipitation strengthening steel. Acta Materialia, 2018. 153: p. 193204.

26. Takahashi, J., Kawakami, K., and Tarui, T., Direct observation of hydrogen-trapping sites in vanadium carbide precipitation steel by atom probe tomography. Scripta Materialia, 2012. 67(2): p. 213-216.

27. Haley, D., et al., Atom probe tomography observation of hydrogen in high-Mn steel and silver charged via an electrolytic route. International Journal of Hydrogen Energy, 2014. 39(23): p. 12221-12229.

28. Kim, Y.J. and Seidman, D.N., Atom-Probe Tomographic Analyses of Hydrogen Interstitial Atoms in Ultrahigh Purity Niobium. Microscopy and Microanalysis, 2015. 21(3): p. 535543.

29. Redhead, P.A., Hydrogen in Vacuum Systems: An Overview, in AIP Conference Proceedings. 2003. p. 243-254. 
30. Sundell, G., Thuvander, M., and Andren, H.O., Hydrogen analysis in APT: Methods to control adsorption and dissociation of H-2. Ultramicroscopy, 2013. 132: p. 285-289.

31. Stephenson, L.T., et al., The Laplace Project: An integrated suite for preparing and transferring atom probe samples under cryogenic and UHV conditions. Plos One, 2018. 13(12).

32. Gong, P., et al., The effect of molybdenum on interphase precipitation and microstructures in microalloyed steels containing titanium and vanadium. Acta Materialia, 2018. 161: p. 374-387.

33. Gault, B., et al., Atom probe microscopy. 2012, New York: Springer.

34. Thuvander, M., et al., Quantitative atom probe analysis of carbides. Ultramicroscopy, 2011. 111(6): p. 604-608.

35. Takahashi, J., Kawakami, K., and Kobayashi, Y., Quantitative analysis of carbon content in cementite in steel by atom probe tomography. Ultramicroscopy, 2011. 111(8): p. 12331238.

36. Marceau, R.K.W., Choi, P., and Raabe, D., Understanding the detection of carbon in austenitic high-Mn steel using atom probe tomography. Ultramicroscopy, 2013. 132: p. 239-247.

37. Bas, P., et al., A General Protocol for the Reconstruction of 3d Atom-Probe Data. Applied Surface Science, 1995. 87-8(1-4): p. 298-304.

38. Briant, C.L., The Effects of Sulfur and Phosphorus on the Intergranular Corrosion of 304 Stainless-Steel. Corrosion, 1980. 36(9): p. 497-509.

39. Kuzmina, M., et al., Linear complexions: Confined chemical and structural states at dislocations. Science, 2015. 349(6252): p. 1080-1083.

40. Sha, W., et al., Some Aspects of Atom-Probe Analysis of Fe-C and Fe-N Systems. Surface Science, 1992. 266(1-3): p. 416-423.

41. Gault, B., et al., Advances in the calibration of atom probe tomographic reconstruction. Journal of Applied Physics, 2009. 105(3).

42. Williams, C.A., et al., Defining clusters in APT reconstructions of ODS steels. Ultramicroscopy, 2013. 132: p. 271-278.

43. Paxton, A.T. and Katzarov, I.H., Quantum and isotope effects on hydrogen diffusion, trapping and escape in iron. Acta Materialia, 2016. 103: p. 71-76.

44. Gerstl, S.S.A. and Wepf, R., Methods in Creating, Transferring, \& Measuring Cryogenic Samples for APT. Microscopy and Microanalysis, 2015. 21(S3): p. 517-518.

45. Yao, L., A filtering method to reveal crystalline patterns from atom probe microscopy desorption maps. Methodsx, 2016. 3: p. 268-273.

46. Geiser, B.P., et al., Spatial distribution maps for atom probe tomography. Microscopy and Microanalysis, 2007. 13(6): p. 437-447.

47. 'libatomprobe', http://apttools.sourceforge.net/ (14 Jul 2017) 


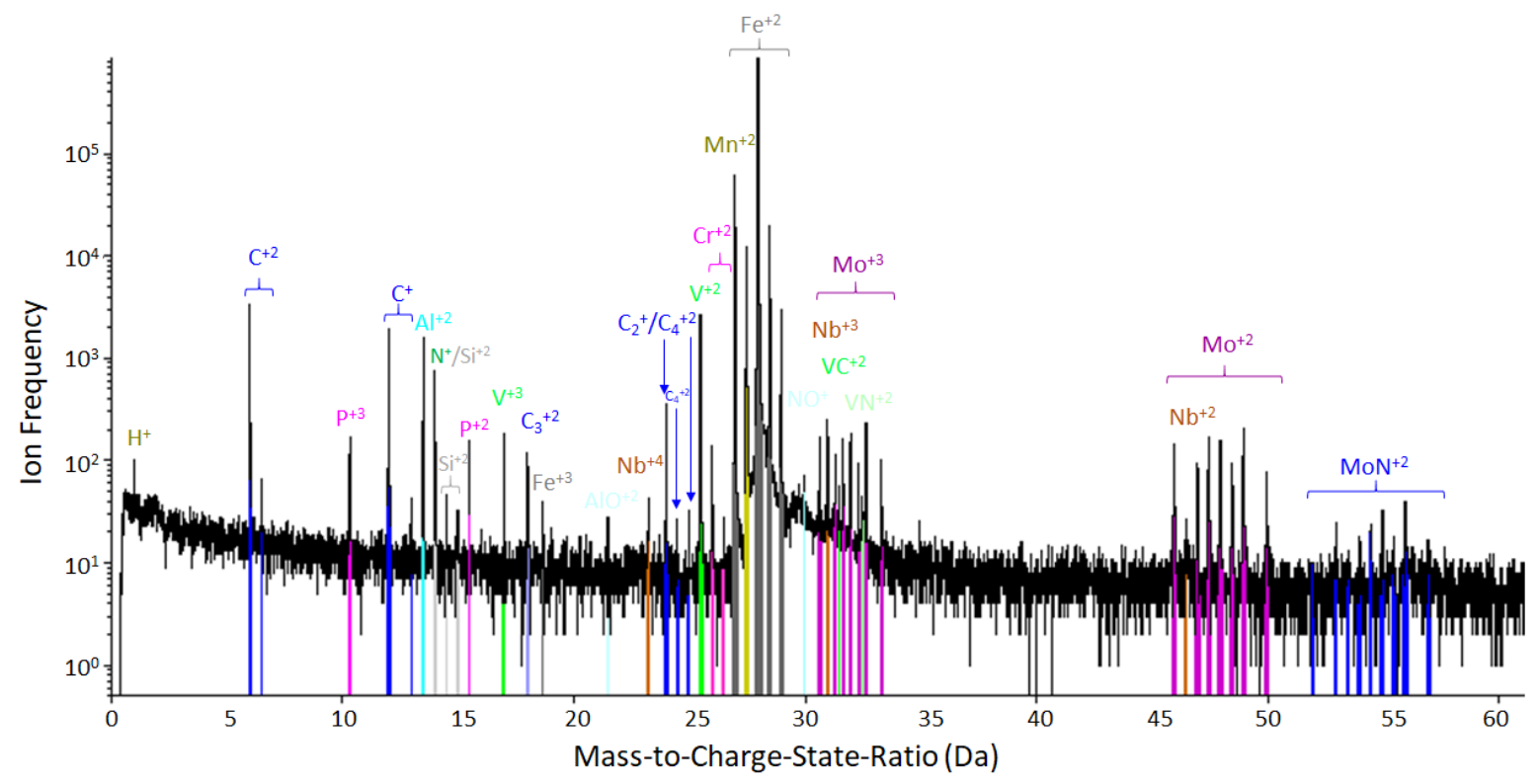

(a)
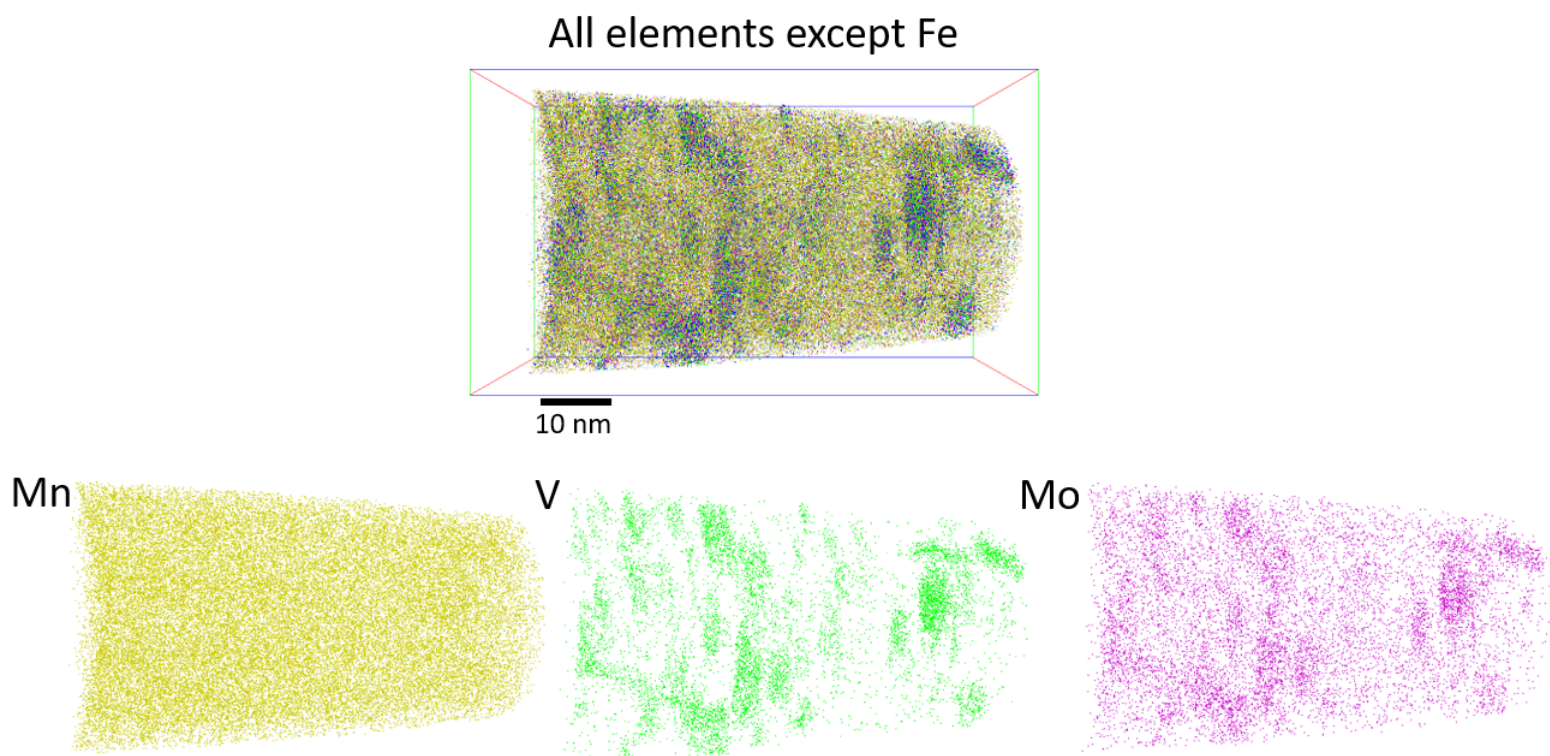

Al

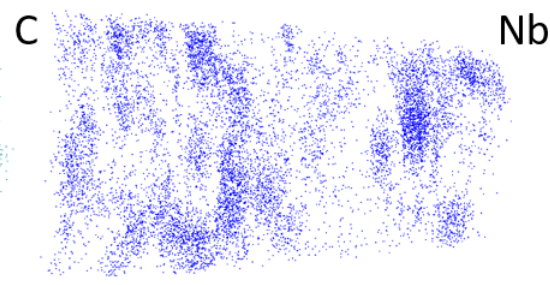

(b)

Figure 1. APT results of uncharged sample (a) mass spectrum and (b) 3D atom maps of primary elements, i.e. solution $\mathrm{Mn}$ and $\mathrm{Al}$ and precipitation $\mathrm{V}, \mathrm{Mo}, \mathrm{C}$, and $\mathrm{Nb}$. Fe is not shown for clarity. 
(a)

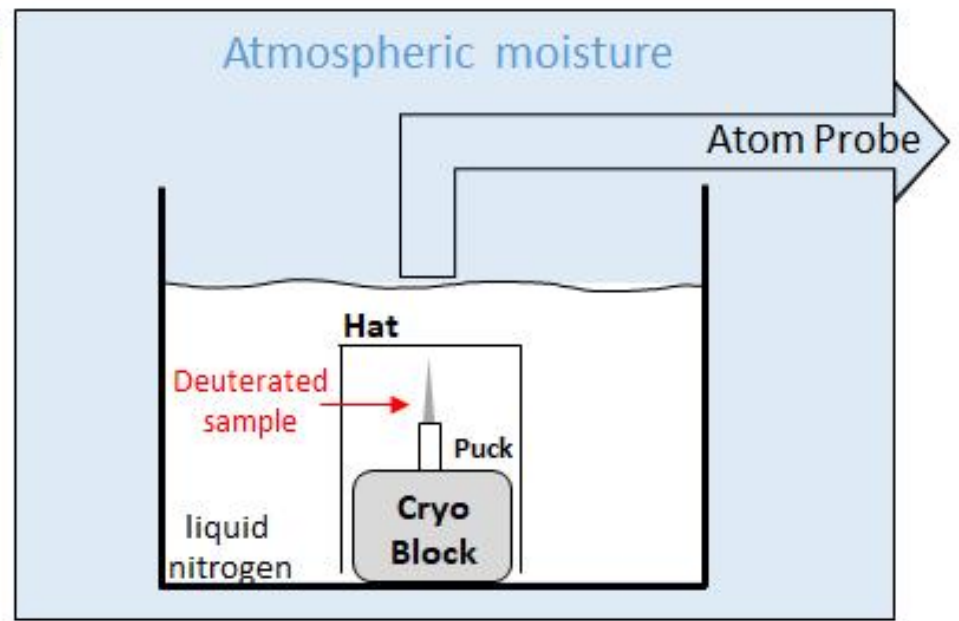

(b)
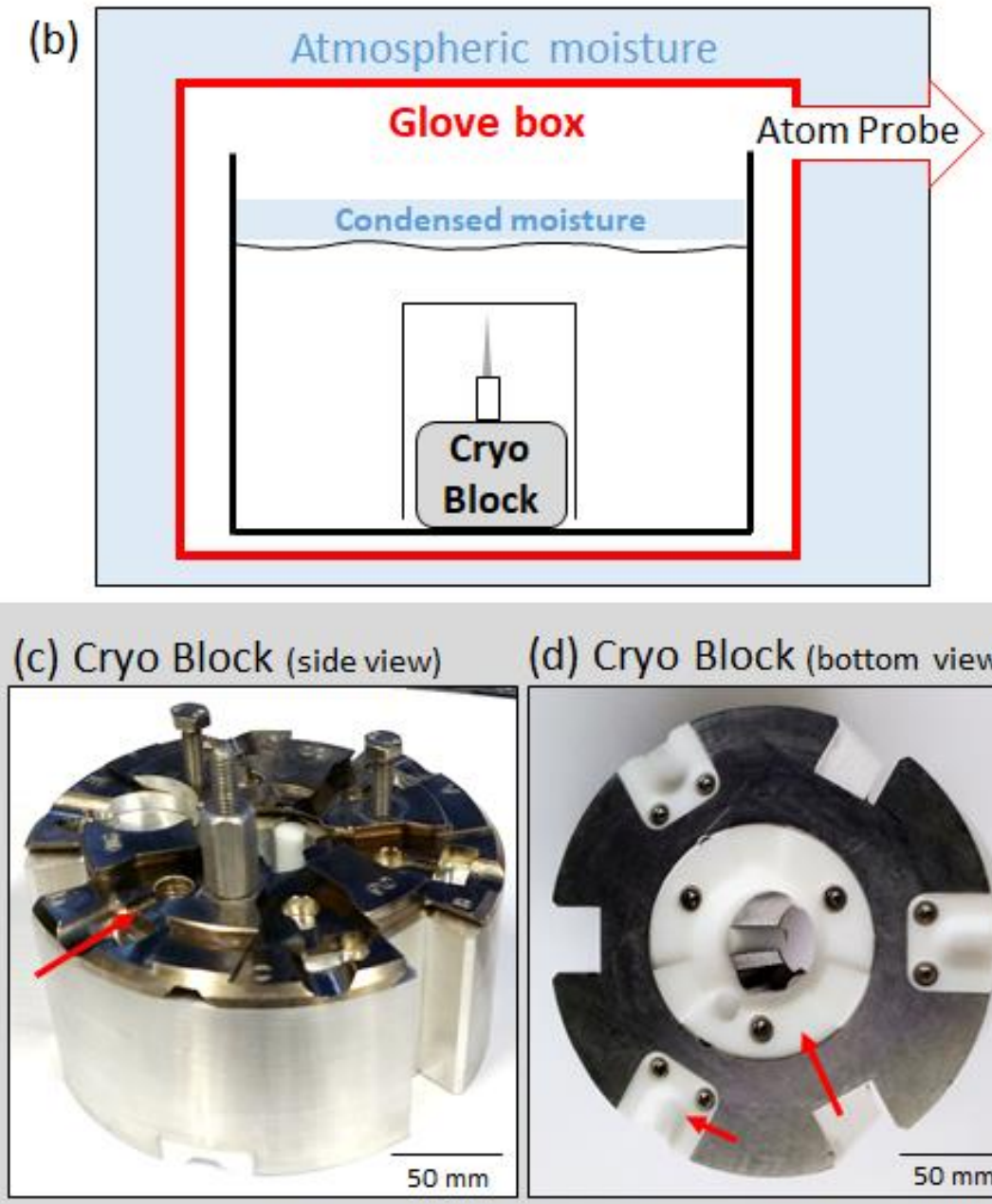

(d) Cryo Block (bottom view)

Figure 2. Schematics of (a) Direct Transfer and (b) Box Transfer. (c) and (d) the side and bottom views of the cryo block (CAMECA). The red arrows in (c) and (d) show the puck loading location and the insulating regions, respectively. 

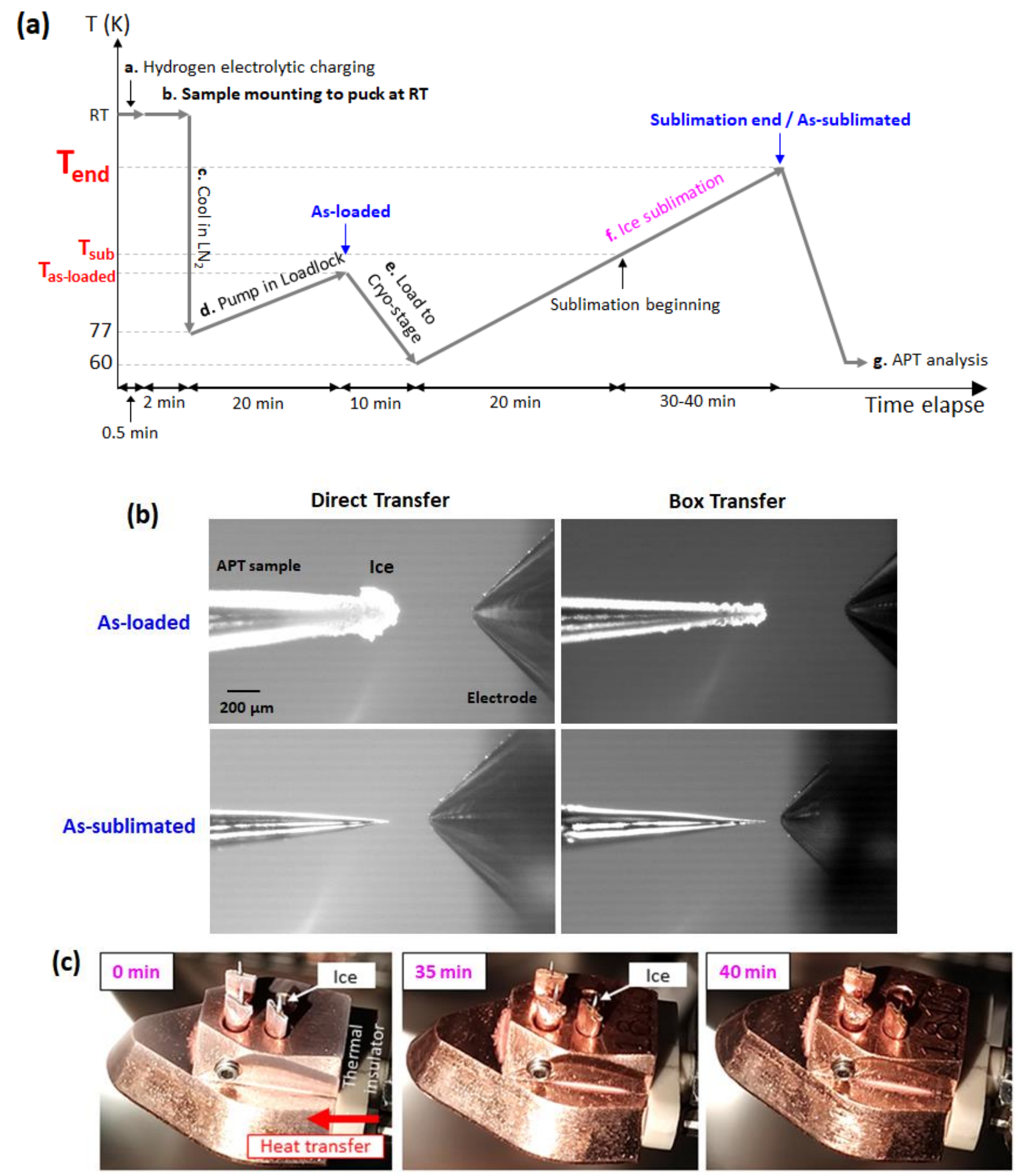

Figure 3. (a) Schematic temperature history of the cryo-sample. (b) The respective tip images before and after the sublimations in the Direct Transfer and Box Transfer. (c) The time-elapsed sample images during the sublimation in the step $f$ of (a). 


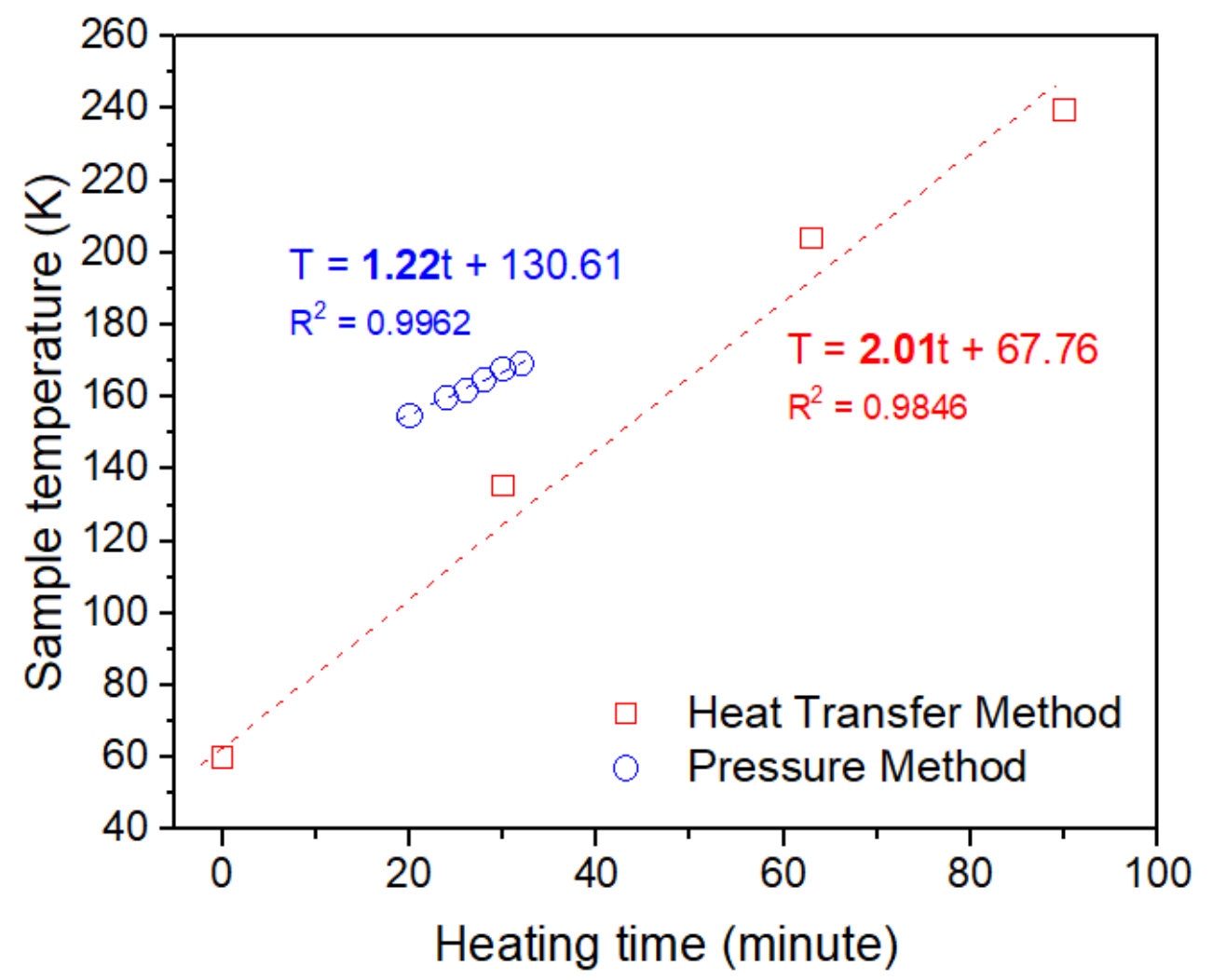

Figure 4. The sample temperatures (T) measured by heat transfer method (red) and pressure method (blue) as a function of the heating time (t) and their respective linear fitting results. 


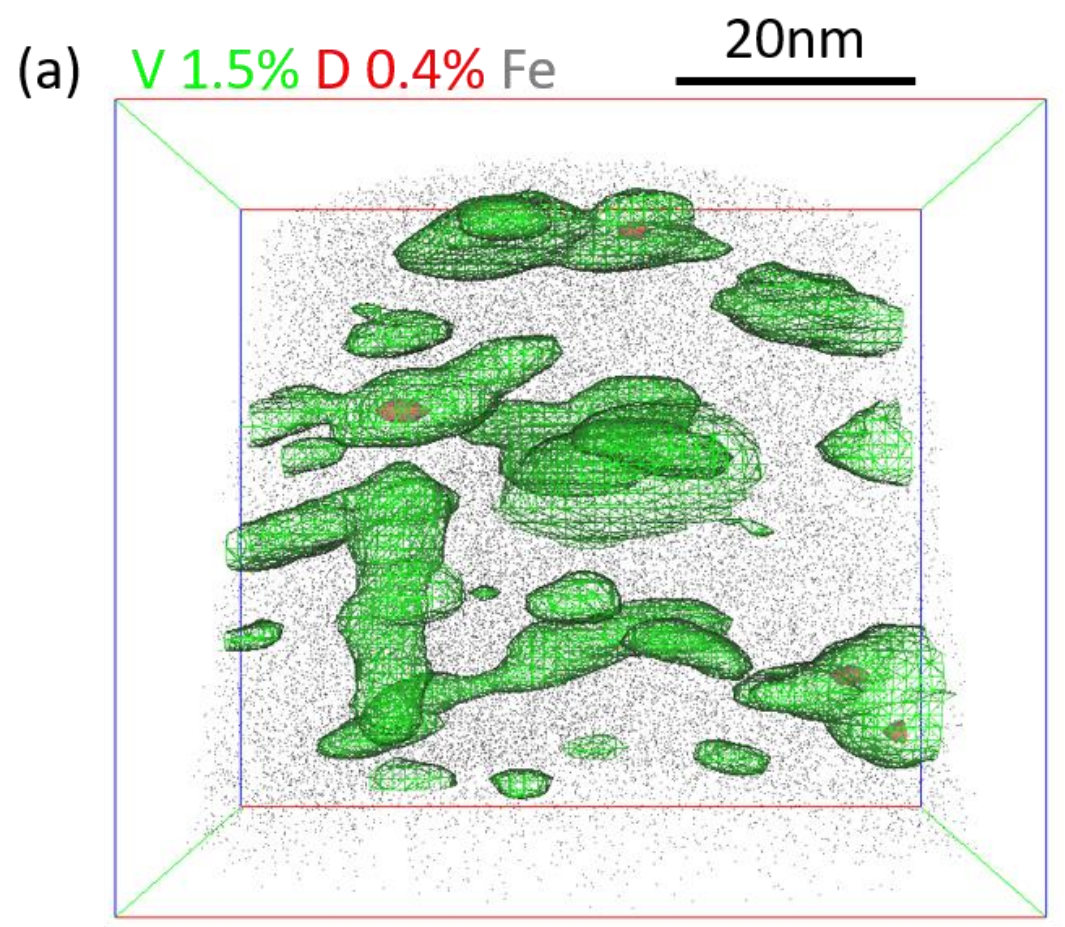

(b)

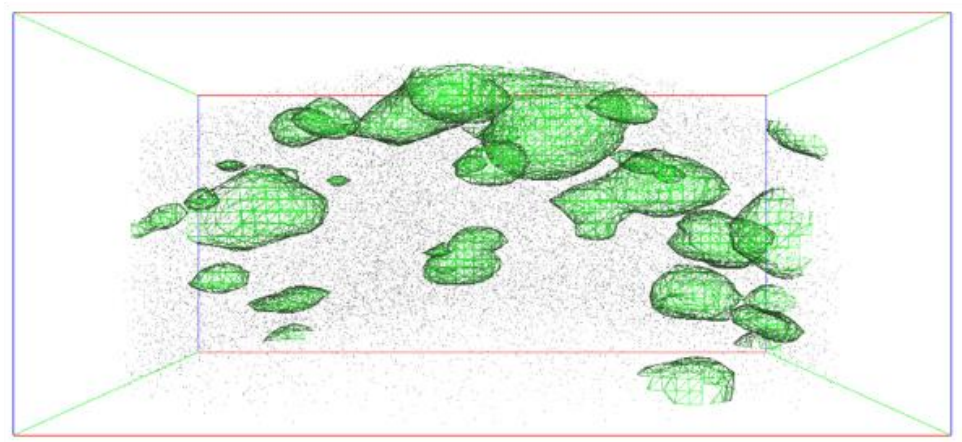

Figure 5. The atom probe reconstructions of (a) a RT Transfer sample and (b) its continuing experiment result after leaving in the buffer chamber for 12 hours to fully desorb charged $\mathrm{D}$. The isoconcentration surfaces of 1.5 at.\% $\mathrm{V}$ and 0.4 at.\% D show the locations of the $\mathrm{V}-\mathrm{Mo}-\mathrm{Nb}$ carbides and the deuterium concentrations. 
(a) $\mathrm{V} 1.5 \% \mathrm{D} 0.4 \% \mathrm{Fe}$

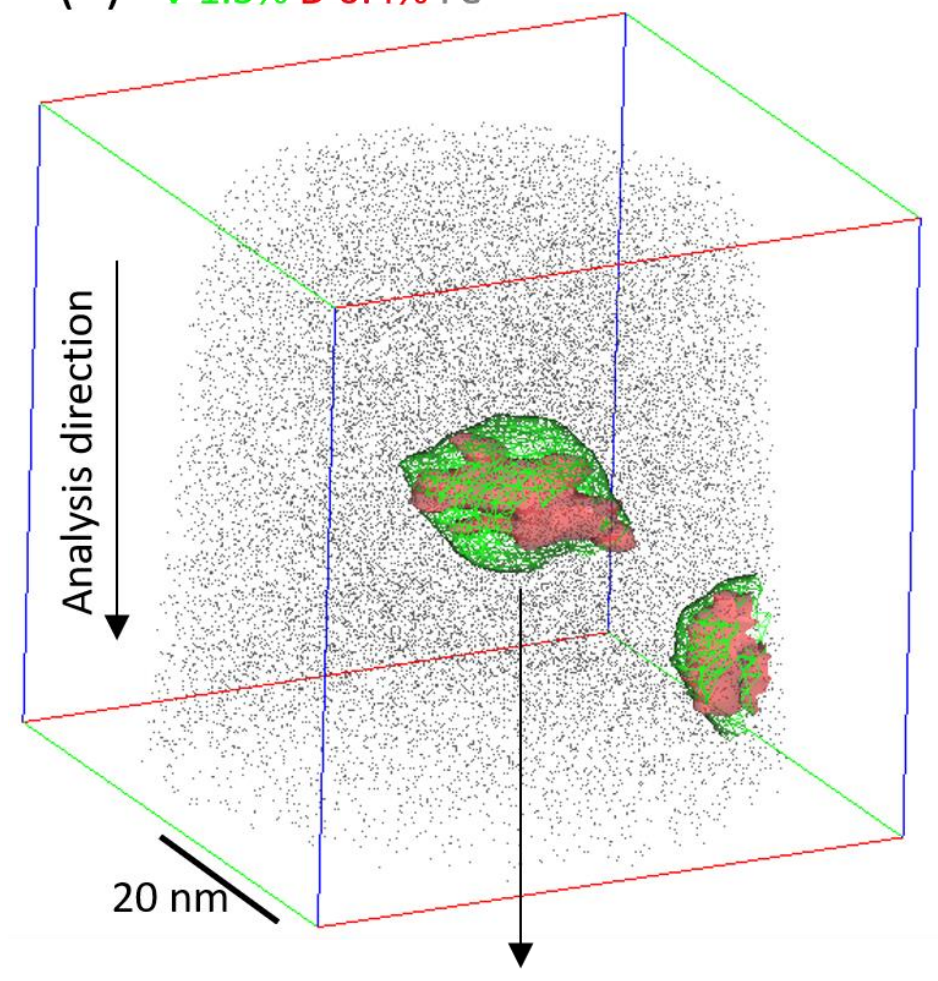

(b) $\mathrm{VDFe}$

$18 \times 13 \times 13 \mathrm{~nm}$

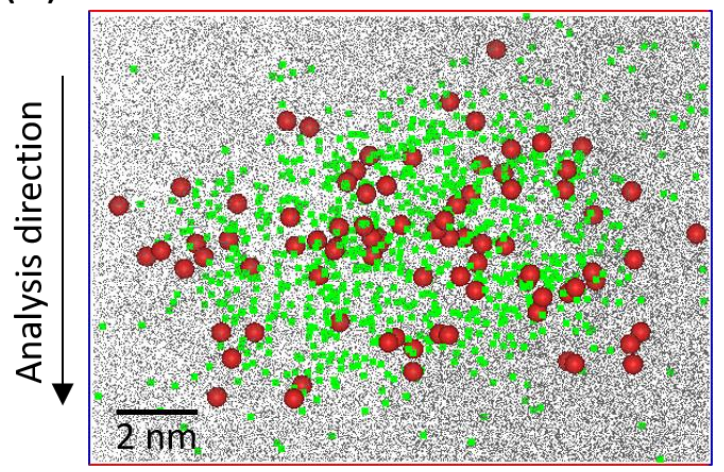

$18 \times 2 \times 13 \mathrm{~nm}$

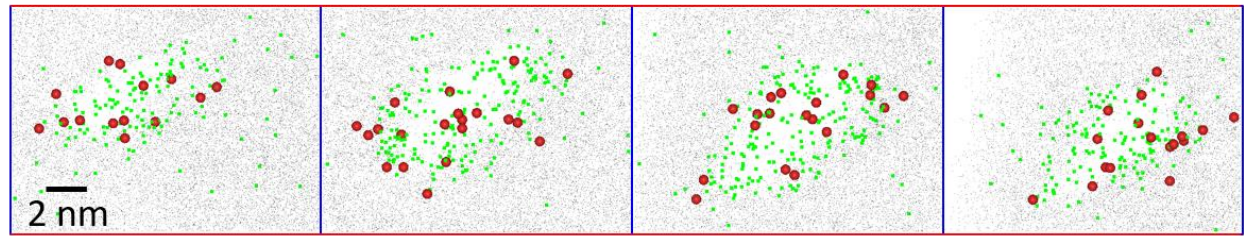

Figure 6. (a) The atom probe isoconcentration surfaces of 1.5 at. $\% \mathrm{~V}$ and 0.4 at. $\% \mathrm{D}$ from a sample using Direct Transfer method, showing the correlated locations of the V-Mo-Nb carbides and the deuterium concentrations. (b) The local view and sliced views of the central carbide ROI in (a). 


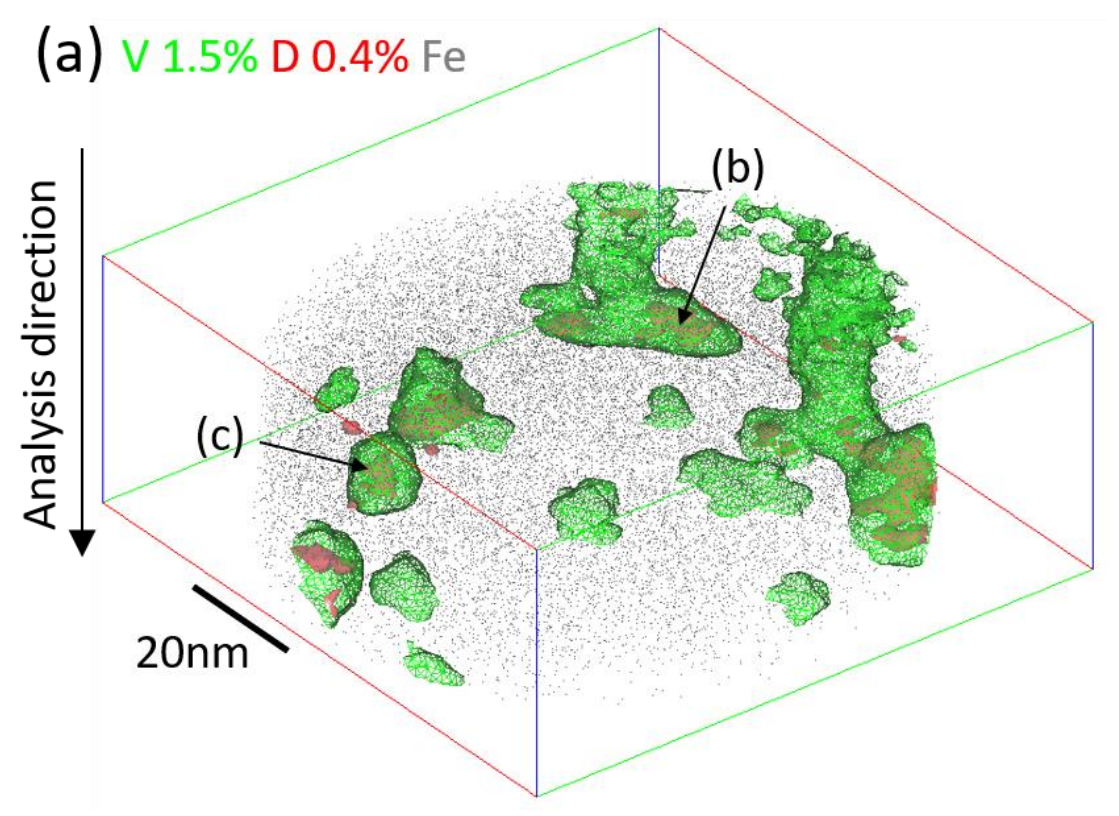

(b) VDFe $15 \times 12 \times 15 \mathrm{~nm}$
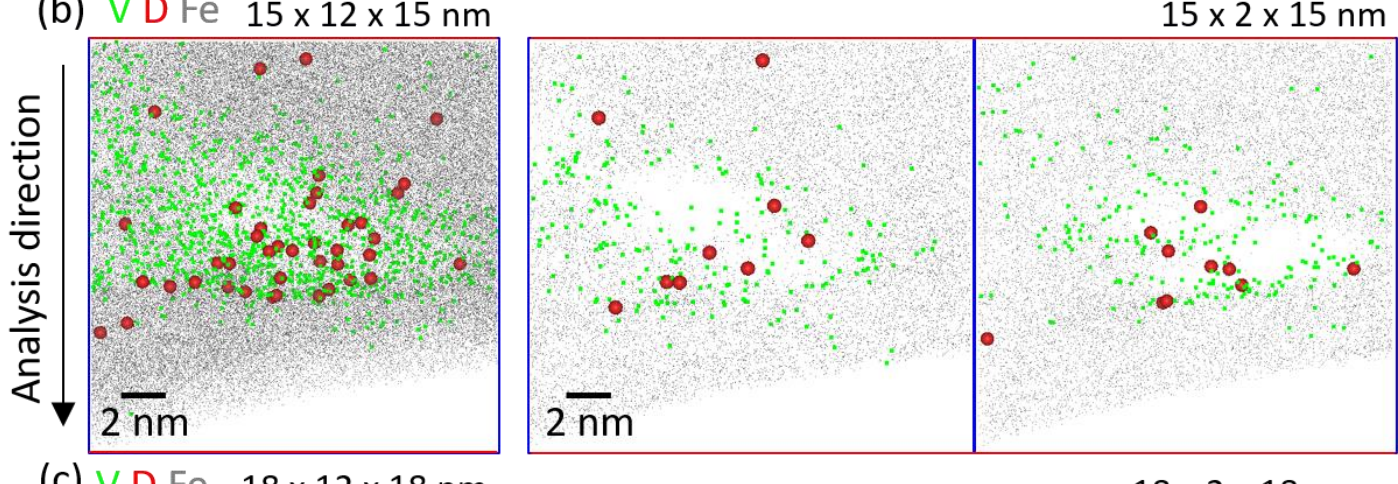

(c) V D Fe $18 \times 12 \times 18 \mathrm{~nm}$
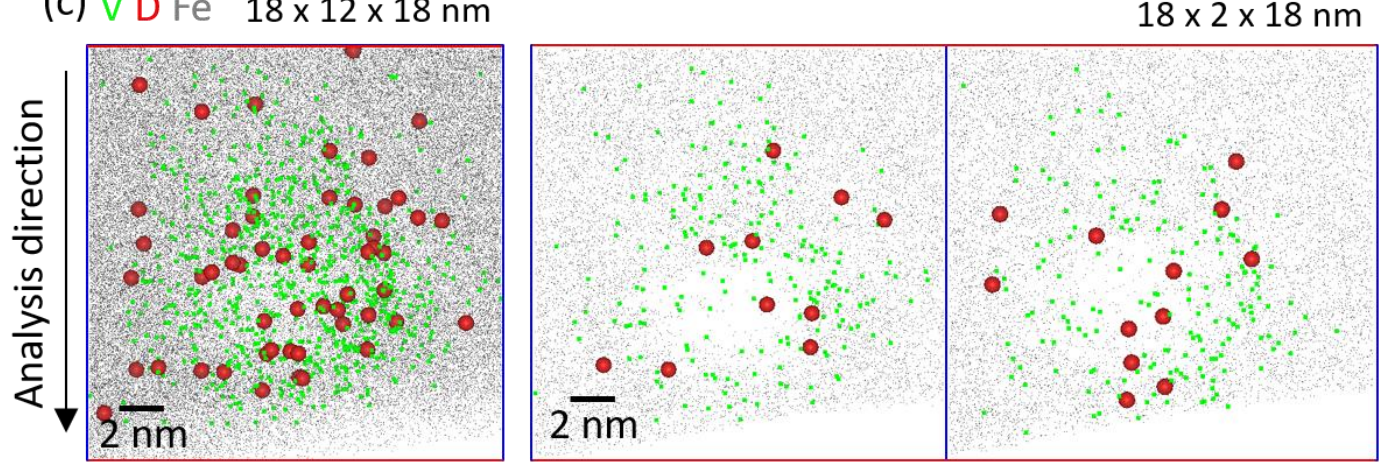

Figure 7. (a) The atom probe isoconcentration surfaces of 1.5 at. $\% \mathrm{~V}$ and 0.4 at. $\% \mathrm{D}$ from a Box Transfer sample, showing the corelated locations of the V-Mo-Nb carbides and the deuterium concentrations. (b) and (c) are the respective $x-y$ plane (note the correspondent coloured axes) sliced views from the centres of two arrowed ROIs in (a). 


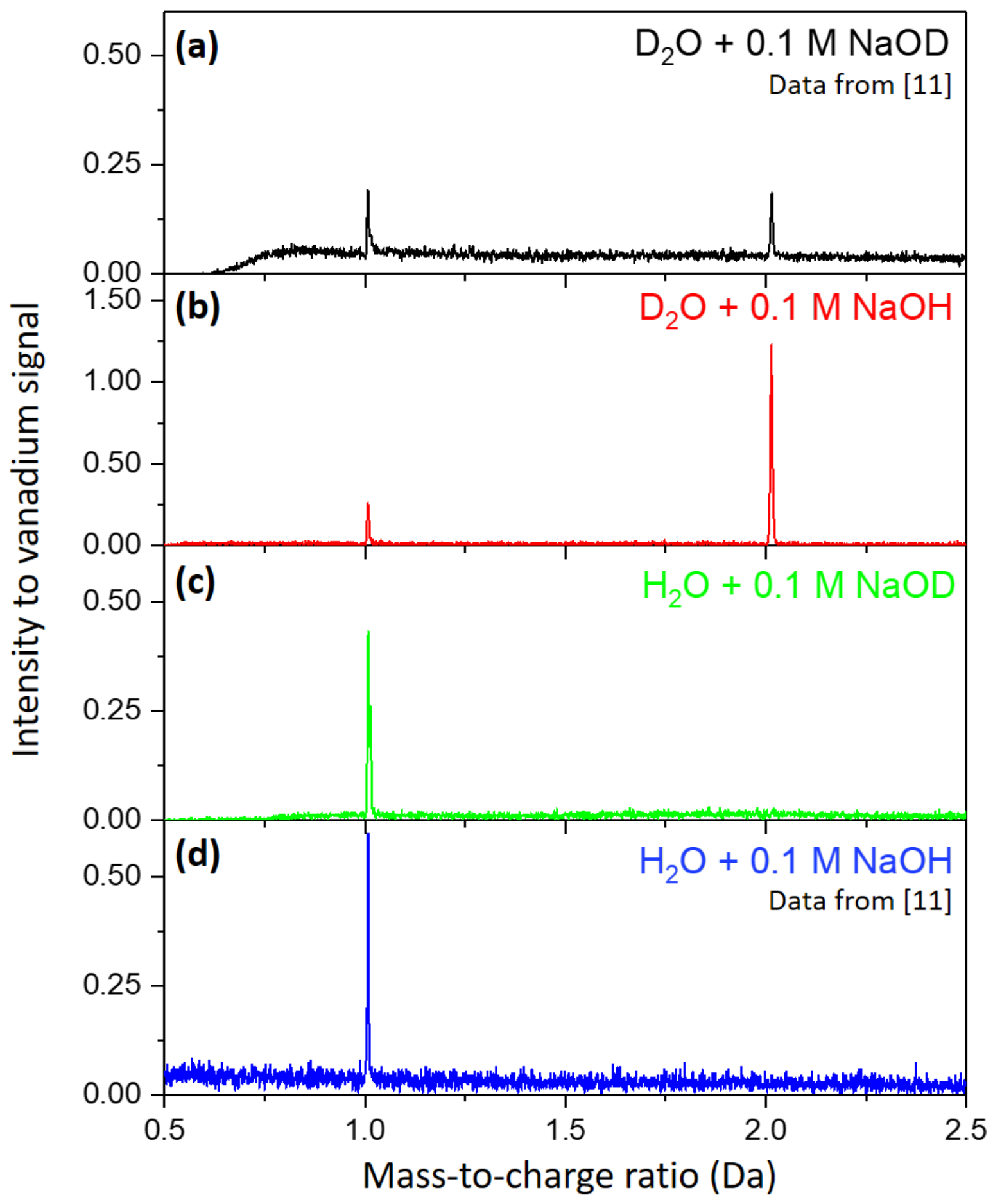

Figure 8 . The uncorrected atom probe mass spectra normalised to their corresponding vanadium peak intensities for the electrolytic hydrogen charging solutions of (a) $\mathrm{D}_{2} \mathrm{O}+0.1 \mathrm{M} \mathrm{NaOD}$, (b) $\mathrm{D}_{2} \mathrm{O}+0.1 \mathrm{M} \mathrm{NaOH}$, (c) $\mathrm{H}_{2} \mathrm{O}+0.1 \mathrm{M} \mathrm{NaOD}$ and (d) $\mathrm{H}_{2} \mathrm{O}+0.1 \mathrm{M} \mathrm{NaOH}$, respectively. 

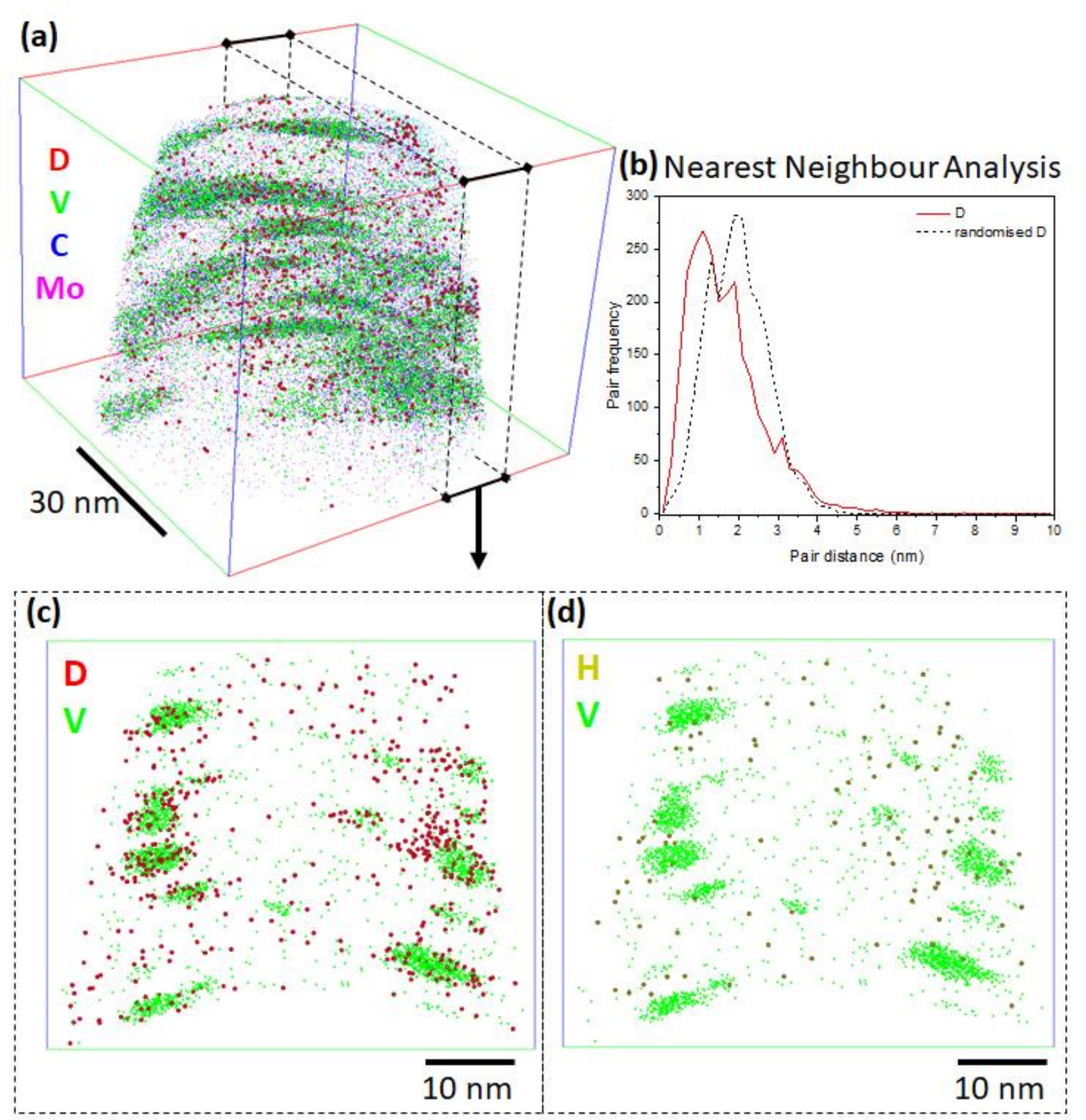

Figure 9. $\mathrm{D}_{2} \mathrm{O}+\mathrm{NaOH}$ charged sample's (a) 3D reconstruction, (b) nearest-neighbour analysis showing the D clustering, (c) a sliced view of the 3D reconstruction showing only D and V and (d) the same slice of (c) showing ${ }^{1} \mathrm{H}$ noise and $\mathrm{V}$ and the absence of their spatial correlation. 


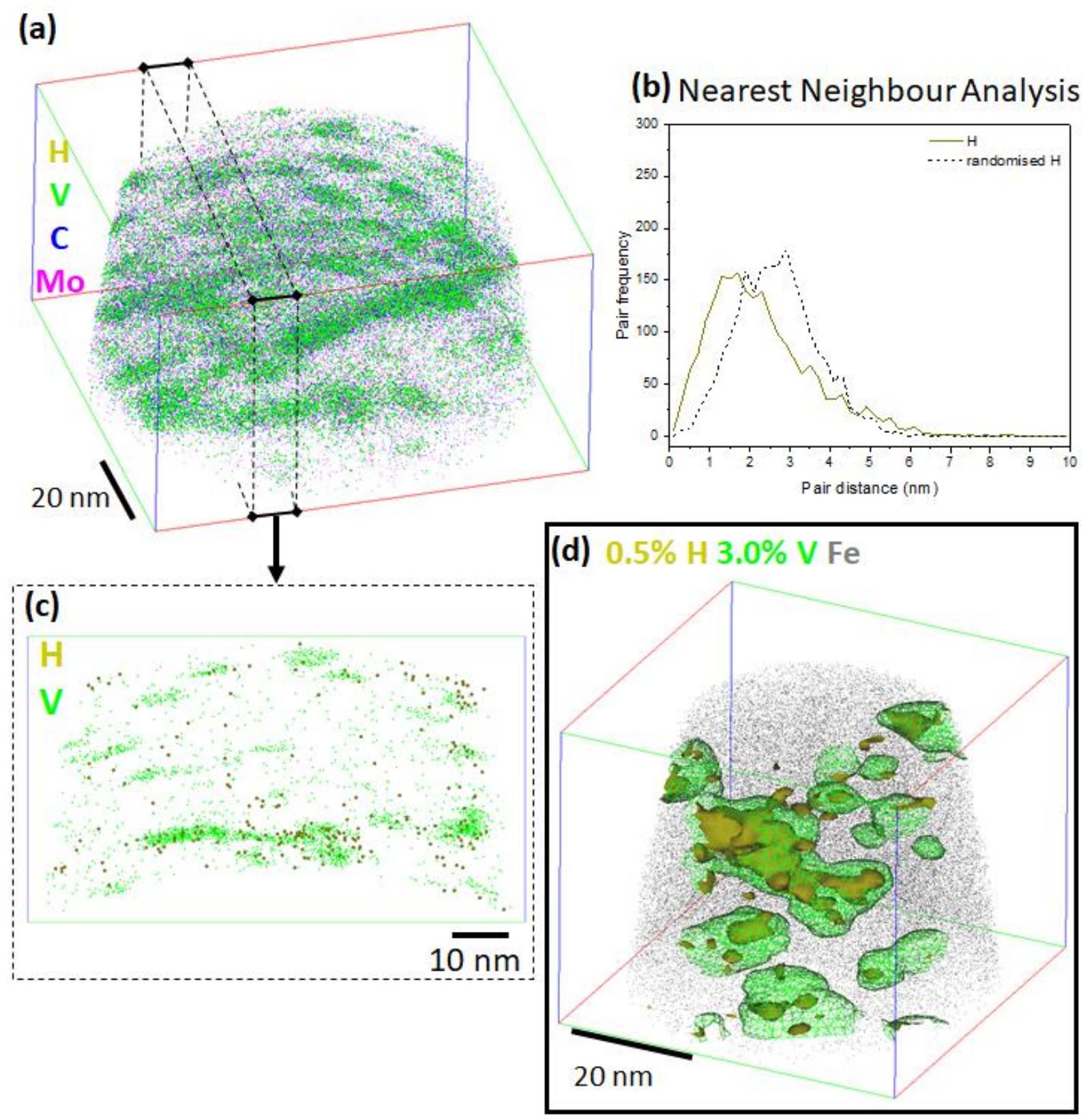

Figure 10. $\mathrm{H}_{2} \mathrm{O}+\mathrm{NaOD}$ charged sample's (a) 3D reconstruction, (b) nearest-neighbour analysis showing the ${ }^{1} \mathrm{H}$ clustering, (c) sliced view of (a) showing only ${ }^{1} \mathrm{H}$ and $\mathrm{V}$, (d) the isosurfaces of 0.5 at. $\%{ }^{1} \mathrm{H}$ and $3 \% \mathrm{~V}$ and iron atoms of $\mathrm{H}_{2} \mathrm{O}+\mathrm{NaOH}$ charged sample from [24], showing the correlation between $\mathrm{H}$ and $\mathrm{V}$ isosurfaces. 
Supplementary section since this page 


\section{S1. Determination of atom probe data reconstruction parameters}

The raw event histogram of an atom probe experiment from the studied steel sample was first extracted from the IVAS as shown in Figure S1(a). Subsequently, the event histogram was processed using the principle specified in [45] and its corresponding computational tool available at [44] for showing only the signals having consecutive events after them. This allowed the poles being differentiated as shown in Figure S1(b) and being identified after comparing with a BCC crystal pole map (Figure S1(c)). With the knowledge of the poles, a spatial distribution map (SDM) [46] (Figure S1(d)) of a 2-nm cylinder along the $\langle 110\rangle$ pole was used to assist correcting the reconstruction parameters until the associated lattice spacing matching the theoretical value of BCC iron as $0.202 \mathrm{~nm}$. And Figure S1(e) of the SDM of a 2-nm cylinder along the <200> pole was used to show the 45-degree atom distribution relationship between the signals along the <110> and $<200>$ poles, confirming the consistency given by the reconstruction inputs in BCC crystal. 


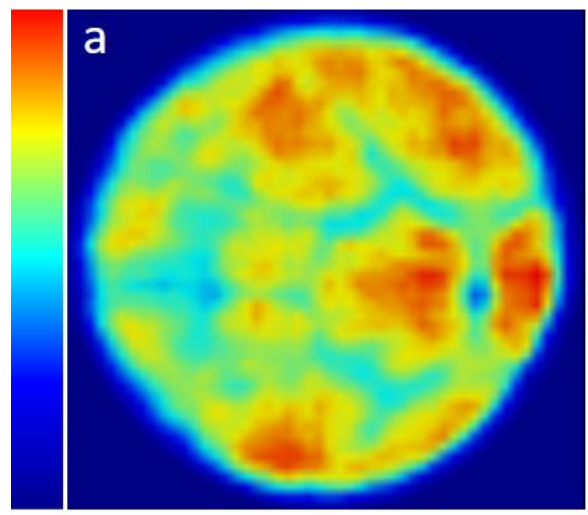

d. 110 planes

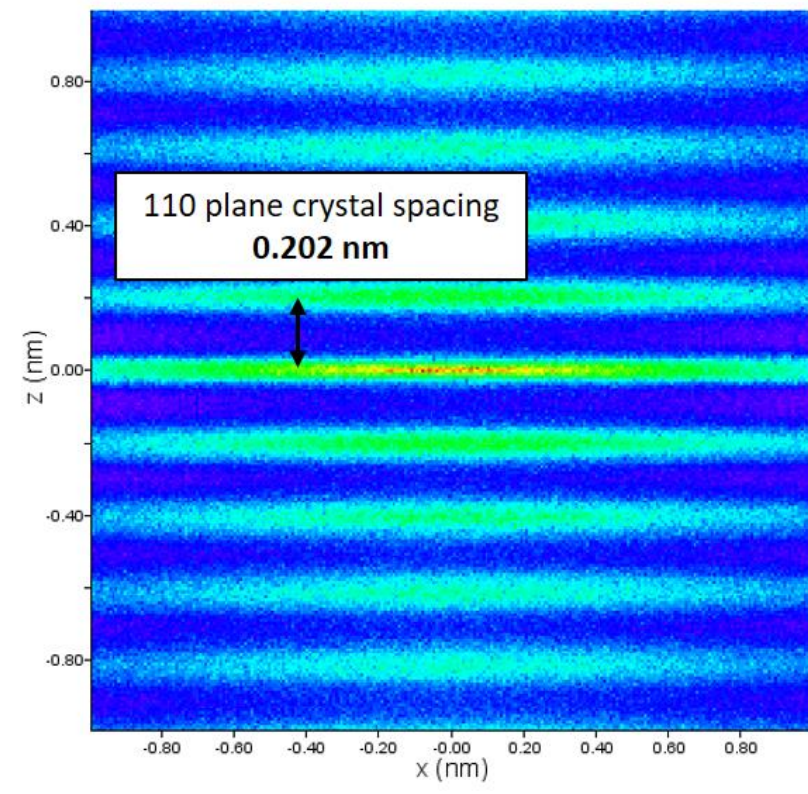

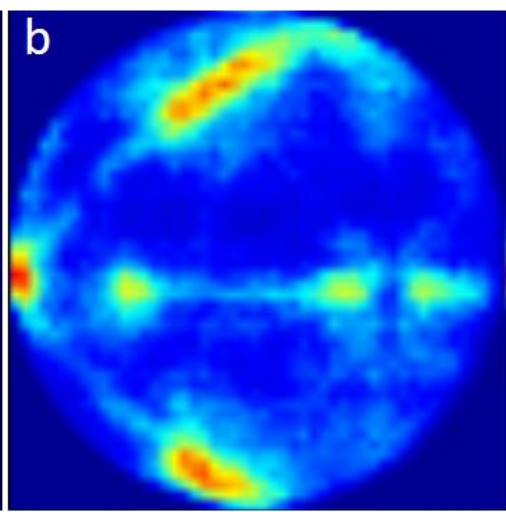

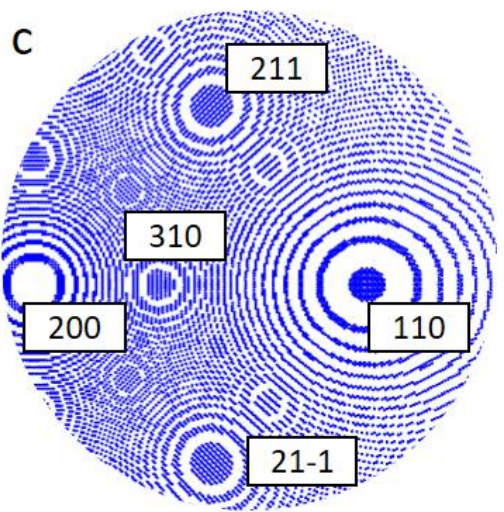

e. 200 planes

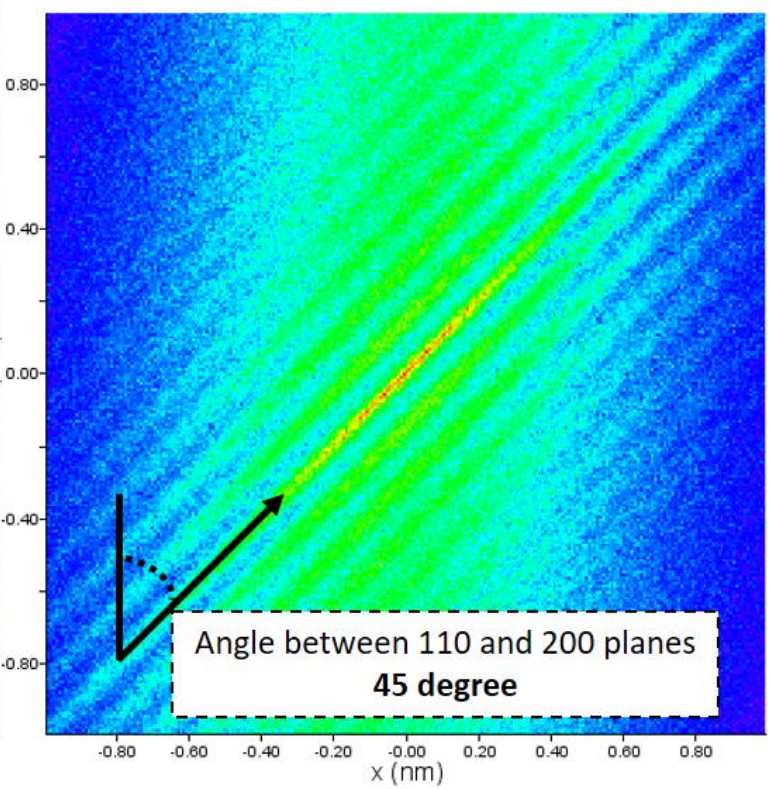

Figure S1. (a) Raw event histogram. (b) The event histogram after the data processing. (c) The BCC crystal pole map corresponding to (b). (d) The spatial distribution map (SDM) of a 2-nm cylinder along the $<110>$ pole. (e) The SDM of a 2-nm cylinder along the $<200>$ pole. 


\section{S2. Determination of the cluster parameters in 3D atom map data}

The cluster parameters used in this studied were defined by the method proposed in [42]. The numbers of identified clusters in the randomised dataset $\left(\mathrm{N}_{\text {rand }}\right)$ and real dataset $\left(\mathrm{N}_{\text {real }}\right)$ were first examined with various minimum number of cluster atoms $\left(\mathrm{N}_{\min }\right)$ as shown in Figure $\mathrm{S} 2(\mathrm{a})$ and (b), respectively. Then the computed results of the object function $\left(\left(\mathrm{N}_{\text {real }}-\mathrm{N}_{\text {rand }}\right) / \mathrm{N}_{\text {real }}\right)$ was plotted against $d_{\max }$, as shown in (c) for the raw plot and in (d) for the magnified view between 0.8 and 1.0. Herein the computed objective function above 0.95 was used for the subsequent cluster analysis verification. A selection of cluster analysis parameters, as the arrows noted in Figure S2(a) and (d), then was fed into IVAS for examining the resulting cluster configurations shown in (e) to (h). Figure S2(e) allows confirming the possible incorporation of artefact with many small clusters even in an artificially randomised data under a problematic parameter selection. Figure S2(f), (g) and (h) show the cluster configurations from using the respective parameter inputs noted in (d). Figure S2(f) shows the discrete clusters highlighted by white arrows, and Figure S2(g) shows a large red joint cluster, which are both regarded as false results. Whereas Figure S2(h) using an intermediate parameter selection $\left(\mathrm{d}_{\max }=1 \mathrm{~nm}\right.$ and $\left.\mathrm{N}_{\min }=100\right)$ noted in $(\mathrm{d})$ resulted in the cluster configuration regarded as relatively reasonable. 

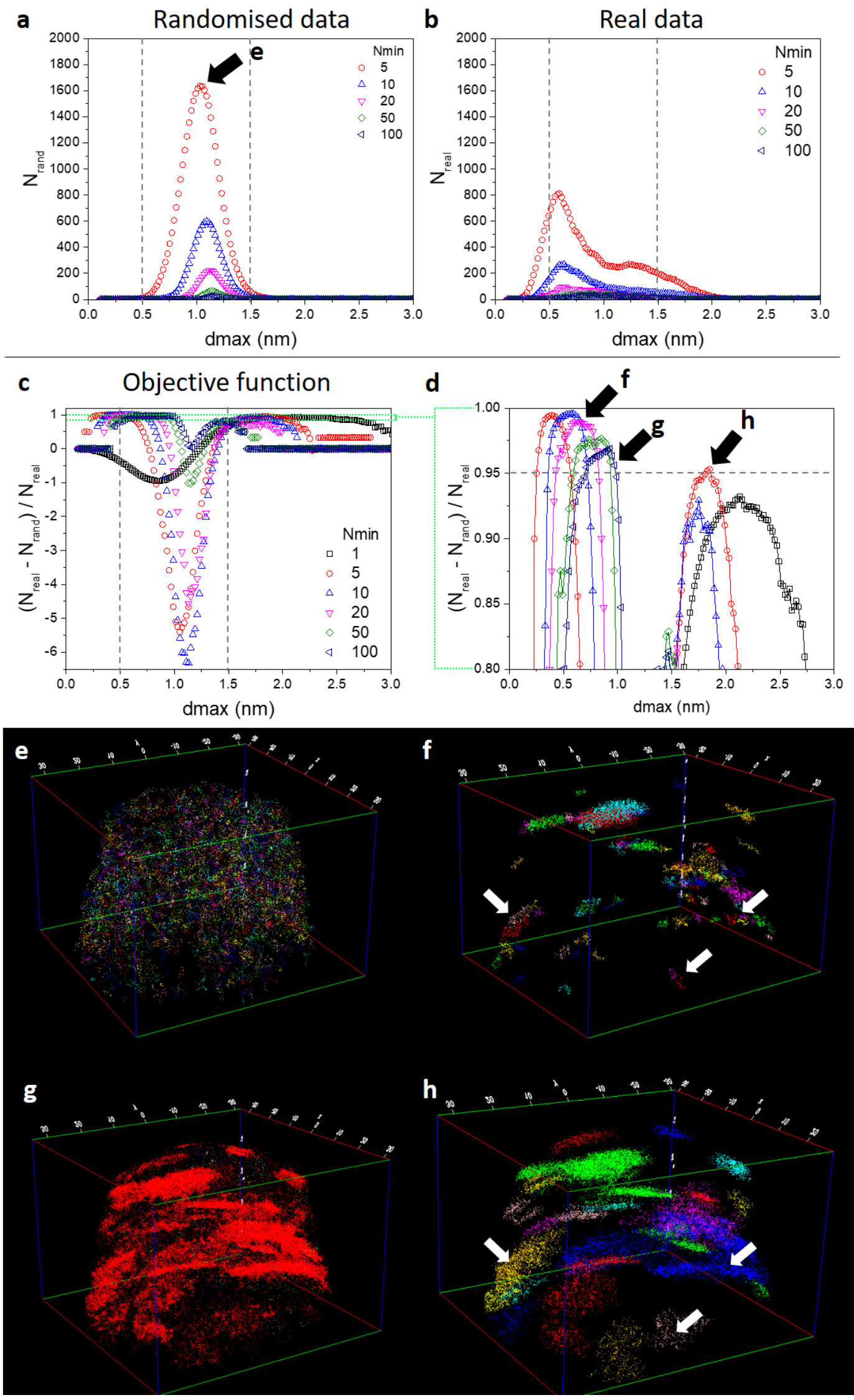
Figure S2. (a) $\mathrm{N}_{\text {rand }}$ as a function of $\mathrm{d}_{\max }$ with various $\mathrm{N}_{\min }$. (b) $\mathrm{N}_{\text {real }}$ as a function of $\mathrm{d}_{\max }$ with various $\mathrm{N}_{\text {min. }}$ (c) Object function $\left(\left(\mathrm{N}_{\text {real }}-\mathrm{N}_{\text {rand }}\right) / \mathrm{N}_{\text {real }}\right)$ as a function of $\mathrm{d}_{\max }$. (d) The magnified view between 0.8 and 1.0 of $(c)$ and the cluster parameters of $\left[\mathrm{d}_{\max }, \mathrm{N}_{\min }\right]$ as $[0.7,20],[1,100]$ and $[1.7,5]$ resulting in (f), (g) and (h), respectively. (f) is the result of $[0.7,20]$ having discrete clusters as highlighted by white arrows. $(\mathrm{g})$ is the result $[1.7,5]$ having a large red joint cluster. And $(\mathrm{h})$ is the cluster configuration from $[1,100]$, which gives the cluster configuration used in this study. 


\section{S3. Procedure of Box Transfer}

A. Prepare

a. A glove-attached plastic bag

b. Approximately 4 litre of $\mathrm{LN}_{2}$

c. Turn off the loadlock turbo pump of LEAP (do not vent air)

B. Connect glove box to the LEAP

C. Cool the cryo-block and the block hat with $\mathrm{LN}_{2}$

D. Conduct hydrogen liquid charging

E. Load the hydrogen-charged samples into the cryo-puck in the $\mathrm{LN}_{2}$

F. Place the samples/cryo-block and the $\mathrm{LN}_{2}$ tank into the glove box

G. Load the cryo-block into the LEAP when relative humidity below $1 \%$

$\mathrm{H}$. Observe the sample condition at the cryo-stage of the analysis chamber

I. Sublimate ice contamination on the samples/cryo-puck in the buffer chamber

J. Load the samples/cryo-puck back to the analysis chamber

K. Commence APT experiment 